\title{
HIV and antiretroviral therapy-related fat alterations
}

John R. Koethe ${ }^{1}$, Claire Lagathu' ${ }^{2}$, Jordan E. Lake ${ }^{3}$, Pere Domingo ${ }^{4}$, Alexandra Calmy 5 , Julian Falutz 6 , Todd T. Brown ${ }^{7}$ and Jacqueline Capeau ${ }^{2 凶}$

Abstract | Early in the HIV epidemic, lipodystrophy, characterized by subcutaneous fat loss (lipoatrophy), with or without central fat accumulation (lipohypertrophy), was recognized as a frequent condition among people living with HIV (PLWH) receiving combination antiretroviral therapy. The subsequent identification of thymidine analogue nucleoside reverse transcriptase inhibitors as the cause of lipoatrophy led to the development of newer antiretroviral agents; however, studies have demonstrated continued abnormalities in fat and/or lipid storage in PLWH treated with newer drugs (including integrase inhibitor-based regimens), with fat gain due to restoration to health in antiretroviral therapy-naive $\mathrm{PLWH}$, which is compounded by the rising rates of obesity. The mechanisms of fat alterations in PLWH are complex, multifactorial and not fully understood, although they are known to result in part from the direct effects of HIV proteins and antiretroviral agents on adipocyte health, genetic factors, increased microbial translocation, changes in the adaptive immune milieu after infection, increased tissue inflammation and accelerated fibrosis. Management includes classical lifestyle alterations with a role for pharmacological therapies and surgery in some patients. Continued fat alterations in PLWH will have an important effect on lifespan, healthspan and quality of life as patients age worldwide, highlighting the need to investigate the critical uncertainties regarding pathophysiology, risk factors and management.

Adipose tissue accounts for $\sim 20-30 \%$ of total body weight in non-obese or overweight persons and for up to $50 \%$ in individuals with obesity and is a primary energy store with important endocrine and immunomodulatory functions. Most adipose tissue depots are comprised of white fat, which is responsible for energy storage and release in response to food intake and energy demand. In humans, brown and beige fat are minor components of adipose tissue and can dissipate energy as heat. White adipocytes sequester dietary lipids in adipose tissue depots to serve as energy stores during periods of fasting and prevent toxic lipid accumulation in other tissues such as muscle, liver, pancreas and heart ${ }^{1}$. In addition, white adipocytes have a role in glucose and lipid metabolism and respond differentially to physiological cues or metabolic stresses by releasing adipokines and lipokines that regulate energy expenditure, appetite control, glucose homeostasis, insulin sensitivity, inflammation, immune function and tissue repair ${ }^{2}$. Although adipose tissue is mainly comprised of adipocytes, it also contains mesenchymal stem cells (which can differentiate into adipocytes, myoblasts, osteoblasts and chondroblasts), immune cells (including $\mathrm{M} 1$ and M2 phenotype macrophages, $\mathrm{CD} 4^{+} \mathrm{T}$ cells and
$\mathrm{CD}^{+} \mathrm{T}$ cells) and fibroblasts, in addition to blood vessels and nerves.

Fat expands owing to excess energy supply either through hypertrophy (increased size of existing adipocytes) or hyperplasia (the formation of new adipocytes from mesenchymal stem cells in adipose tissue). In obesity, hypertrophic adipocytes have different biochemical properties to smaller adipocytes, including increased lipolysis, inflammatory cytokine production and reduced secretion of anti-inflammatory adipokines, such as adiponectin, that are also involved in insulin sensitivity ${ }^{1}$. Such alterations in fat depots predispose to ectopic lipid accumulation and the development of comorbidities, including type 2 diabetes mellitus (T2DM), non-alcoholic fatty liver disease (NAFLD), sarcopenia, atherosclerosis and heart failure ${ }^{2}$. During physiological ageing, fat is lost from lower limbs and increases in the trunk, alongside accumulation of senescent adipocytes that have altered adipogenic potential and increased secretion of proinflammatory cytokines ${ }^{3}$.

Fat alteration became a major concern in Western countries in the mid-1990s, when people living with HIV (PLWH) receiving combination antiretroviral therapy 


\author{
Author addresses \\ ${ }^{1}$ Division of Infectious Diseases, Vanderbilt University Medical Center, Nashville, TN, USA. \\ ${ }^{2}$ Sorbonne Université, Faculty of Medicine, Inserm UMR_S938, Saint-Antoine Research \\ Centre, FRM EQU201903007868, ICAN, RHU CARMMA, Paris, France. \\ ${ }^{3}$ Department of Medicine, University of Texas Health Science Center at Houston, Houston, \\ TX, USA. \\ ${ }^{4}$ Department of Infectious Diseases, HIV Infection Unit, Hospital de la Santa Creu \\ i Sant Pau, Barcelona, Spain. \\ ${ }^{5}$ HIV/AIDS Unit and LIPO \& Metabolism Group, Infectious Disease Division, Geneva \\ University Hospitals, Geneva, Switzerland. \\ ${ }^{6}$ Department of Medicine McGill University Health Center, Montreal, Québec, Canada. \\ ${ }^{7}$ Division of Endocrinology, Diabetes, \& Metabolism, Johns Hopkins University, Baltimore, \\ MD, USA.
}

(cART) developed a condition of severe subcutaneous lipoatrophy (fat loss), which was termed lipodystrophy $y^{4}$. Some of these PLWH with lipodystrophy also had fat gain in the trunk and neck. Historically, these conditions were common in PLWH who were treated with older-generation thymidine analogue nucleoside reverse transcriptase inhibitors (NRTIs; such as zidovudine and stavudine $)^{5}$ (BOX 1) and early protease inhibitors (PIs; such as indinavir and full-dose ritonavir). The toxic effects of thymidine analogue NRTIs on adipocytes led to the development of other antiretroviral agents with a lower risk of severe lipoatrophy, such as the NRTIs tenofovir disoproxil fumarate (TDF) and emtricitabine (also known as FTC). However, more recent studies have demonstrated fat alterations, mainly fat gain and changes in lipid trafficking and storage in individuals using these therapies, which are different from lipodystrophy and are compounded by the rising worldwide prevalence of obesity $^{6}$. Moreover, first-generation thymidine analogue NRTIs can exert long-lasting effects, and fat alterations and metabolic dysregulation can be observed several years after regimen change ${ }^{7}$.

ART initiation and long-term treatment have been almost universally associated with weight and generalized fat gain, particularly in the trunk, leading to abdominal obesity (also known as visceral obesity) ${ }^{8,9}$. Moreover, integrase strand transfer inhibitors (INSTIs) have been associated with weight gain and generalized fat gain in some $\mathrm{PLWH}^{10}$. The NRTI backbone agents used in these patients can also affect weight gain to varying degrees; TDF has been shown to result in less weight gain than other NRTIs in some studies ${ }^{10,11}$, whereas tenofovir alafenamide (TAF; a newer formulation of tenofovir) has been associated with greater weight gain than TDF, abacavir or the non-NRTI (NNRTI) efavirenz in trials ${ }^{11,12}$.

Mechanisms of fat alterations in PLWH are complex and result from the direct effects of HIV proteins and antiretroviral agents on adipocyte health, in addition to changes in microbial translocation and the immune response, increased tissue inflammation and accelerated fibrosis, although these mechanisms are only partly understood. In addition, the effects of HIV and antiretroviral therapies on fat differ between individuals. To this end, a central fat distribution, in particular within the visceral adipose tissue (VAT) depot (an 'apple' shape or android distribution) is generally associated with a less favourable metabolic phenotype, greater inflammation and insulin resistance, and an increased risk of T2DM and cardiovascular diseases ${ }^{13}$. By contrast, a distribution where the subcutaneous adipose tissue (SAT) depot is preferentially expanded, particularly in the lower extremities ('pear' shape or gynoid distribution), is associated with a more favourable metabolic phenotype and insulin sensitivity ${ }^{13}$.

Fat alterations remain an issue in patients receiving contemporary cART, compounded by the rising rates of obesity globally, and particularly in North America. This Primer summarizes the characteristics and mechanisms of HIV-related and ART-related fat alterations, focusing on the current cART era, and discusses the management and critical research gaps in the field.

\section{Epidemiology \\ HIV-associated wasting}

Altered body composition in ART-naive PLWH before the onset of severe $\mathrm{CD} 4^{+} \mathrm{T}$ cell depletion was recognized early in the HIV epidemic and was characterized by lower lean and fat mass than people without HIV. In addition, wasting of lean muscle mass and fat was a hallmark of AIDS early in the epidemic ${ }^{14,15}$. In these early cases, wasting was defined by a weight loss of $>10 \%$ of pre-illness maximum weight or weight at $<90 \%$ of ideal body weight for the patient's gender ${ }^{16,17}$. In men with AIDS, the wasting syndrome was characterized by a disproportionate decrease in lean body mass with relative fat sparing, whereas women with AIDS had a progressive and disproportionate decrease in body fat relative to lean body mass at all severities of wasting ${ }^{18}$.

Wasting in PLWH was primarily caused by a profound caloric deficit, engendered by reduced energy absorption from gastrointestinal barrier dysfunction, opportunistic infections, reduced gastrointestinal transit time (leading to diarrhoea), increased protein turnover (leading to higher catabolism and impaired anabolism) and increased energy requirements ${ }^{19,20}$. This negative energy balance reduced adipocyte lipid content and size, rather than damaging adipocyte bioenergetics or altering the stromal vascular fraction. As such, the mechanisms of this wasting are considered more similar to those of starvation than later descriptions of lipoatrophy early in the cART era. However, prior wasting disease was not without consequences. In ART-naive patients, peripheral fat reductions were generally greater in women than in men ${ }^{21,22}$ and ART-naive women with prior AIDS-defining illnesses (infections and cancers directly associated with AIDS-related immunodeficiency) had less fat than women without such illnesses ${ }^{22}$

\section{cART-related lipoatrophy and lipohypertrophy}

In contrast to wasting, the clinical syndrome of lipoatrophy in patients receiving cART results from damage to adipocytes and stromal vascular cells. The lipoatrophy phenotype was first identified in PLWH who received cART triple-drug therapy, including one first generation PI and two NRTIs (either stavudine or zidovudine $)^{23}$. A high proportion of these patients, up to $80 \%$ in some surveys, reported a perceived striking and stigmatizing change in physical appearance during 


\section{Box 1 Antiretroviral drugs}

The most commonly previously or presently used drugs are indicated below.

Nucleoside or nucleotide reverse transcriptase inhibitors

- Zidovudine (ZDV), also known as azidothymidine (AZT)

- Didanosine (DDI)

- Stavudine (D4T)

- Lamivudine (3TC)

- Abacavir (ABC)

- Tenofovir disoproxil fumarate (TDF)

- Tenofovir alafenamide (TAF)

- Emtricitabine (FTC)

Non-nucleoside reverse transcriptase inhibitors

- Nevirapine (NVP)

- Efavirenz (EFV)

- Etravirine (ETR)
- Rilpivirine (RPV)

- Doravirine (DOR)

Protease inhibitors

- Saquinavir (SQV)

- Indinavir (IDV)

- Ritonavir (RTV)

- Nelfinavir (NFV)

- Lopinavir (LPV)

- Atazanavir (ATV)

- Darunavir (DRV)

Integrase strand transfer inhibitors

- Raltegravir (RAL)

- Dolutegravir (DTG)

- Elvitegravir (EVG)

- Bictegravir (BIC)

aDenotes first generation antiretroviral molecules that were approved by the FDA between 1987 and 1998.

ART use at the same time that $\mathrm{CD} 4^{+} \mathrm{T}$ cell count and survival increased ${ }^{24}$.

In some patients, lipoatrophy was observed in association with truncal fat accumulation. As previously mentioned, many early studies combined the two distinct phenomena of lipoatrophy and lipohypertrophy into a single 'lipodystrophy' phenotype, characterized by broad regional changes (generally reduced limb diameter and facial wasting with or without abdominal or truncal enlargement $)^{25,26}$. However, problems with this mixed definition include the conflation of two distinct syndromes, a reliance on relative proportions (for example, reductions in limb diameter suggest increased abdominal girth) and a lack of objective metrics. An objective definition of the mixed lipodystrophy phenotype, based on dual X-ray absorptiometry (DXA)-derived fat ratios, has not been validated for use in clinical practice ${ }^{4,27}$. Thus, most prevalence studies are based on clinical evaluations of a patient's appearance using semiquantitative clinical scores such as the Lipodystrophy Severity Grading Scale ${ }^{4}$.

Many earlier surveys assessed a combined phenotype of lipoatrophy and/or lipohypertrophy in PLWH and estimated a worldwide prevalence of $13-70 \%$ from the mid-1990s to the early $2000 \mathrm{~s}^{28}$. Patient ethnicity did not appear to markedly influence the prevalence in PLWH, despite differences in susceptibility to VAT accumulation in the general population ${ }^{29,30}$. In African countries/regions, lipoatrophy and/or lipohypertrophy were reported in $\sim 25 \%$ of patients using ART, but prevalence ranged from $1-84 \%^{31}$, with higher values reported in women than in men, and in persons $>40$ years of age than in younger persons ${ }^{32}$. In the Asia-Pacific region, a severe phenotype, defined as disfiguring or obvious body shape changes on casual visual inspection, was reported in $\sim 10 \%$ of PLWH using cART and was strongly associated with the use of stavudine and older PIs ${ }^{33}$.

\section{Lipoatrophy}

Lipoatrophy prevalence has varied over time and geographical location owing to the improved knowledge of risk factors by HIV caregivers and the introduction of newer, less fat-toxic antiretroviral therapies. In general, a loss of $\geq 30 \%$ limb fat (evaluated using DXA) is required for lipoatrophy to become clinically evident ${ }^{34}$ and early or subtle forms can be easily overlooked. Diagnosis is easier in women (who have higher initial amounts of limb fat) than in men (who can have minimal limb fat $)^{35}$. A prospective study and a clinical trial that delineated the natural history of lipoatrophy in PLWH treated with early thymidine analogue-containing cART reported a biphasic trend, with a general fat gain during the first 24-32 weeks after starting cART, in what is viewed as a 'return to health' phenomenon, followed by a median limb fat loss of $13.7 \%$ per year, which varied from $1.7 \%$ per year for zidovudine-lamivudine to $19 \%$ per year for stavudine-didanosine ${ }^{36,37}$. The association of thymidine analogue NRTIs with lipoatrophy was further supported by its partial or total reversion when these drugs were replaced by less toxic NRTIs in some patients ${ }^{38}$. In clinical trials of newer antiretroviral agents, the threshold of limb fat loss for the diagnosis of lipoatrophy is generally $10-20 \%$ and prevalence after 3 years of therapy is often $5-10 \%$ depending on the type or class of antiretroviral ${ }^{39}$

Owing to the high risk of lipoatrophy with thymidine analogue NRTIs, the WHO issued a statement in 2009 that recommends the avoidance of stavudine for HIV treatment ${ }^{40}$. With the near-universal switch to TDF-based regimens in low-income countries, it is expected that the prevalence of lipoatrophy will decrease worldwide as older drug regimens are replaced with less toxic alternatives. However, despite the avoidance of both zidovudine and stavudine ${ }^{38}$, the prevalence of lipoatrophy is still high among many long-term ART recipients owing to surviving historical cases ${ }^{28}$.

Indeed, individuals who received thymidine analogue NRTIs as part of older cART regimens often have persistent fat alterations several years after therapy discontinuation ${ }^{41,42}$. Long-term infected (median duration 12.2 years) and cART-treated PLWH in the AGEhIV cohort had higher waist and lower hip circumferences than people without HIV with similar demographic and behavioural characteristics ${ }^{43}$. In addition, cumulative prior exposure to stavudine, zidovudine or didanosine was associated with persistent CT evidence of decreased SAT and increased abdominal VAT in the COCOMO study as well as a higher risk of hypertension, high total cholesterol and low HDL cholesterol $^{44,45}$. The increased VAT was greater with longer cumulative exposure to thymidine analogue NRTIs or didanosine and was observed at all time points after discontinuation of the drugs ${ }^{44}$. Moreover, $22 \%$ patients from the APROCO cohort who were previously treated with thymidine NRTIs have persistent mixed lipoatrophy and/or lipohypertrophy and high rates of insulin resistance ${ }^{46}$. Young adults who were infected with HIV during childhood and exposed to older-generation NRTIs are particularly susceptible to persistent lipoatrophy after discontinuation and have 
a higher prevalence of fat alteration than age-paired persons from the general population ${ }^{38,47}$.

Aside from the use of older NRTIs, other risk factors associated with lipoatrophy include HIV-related factors, such as a low $\mathrm{CD} 4^{+} \mathrm{T}$ cell count (generally $<100$ cells $/ \mathrm{mm}^{3}$ ) and high plasma HIV1 RNA levels $\left(>10^{5}\right.$ copies $\left./ \mathrm{ml}\right)$ at cART initiation ${ }^{28}$. For host-related factors, such as age, cut-offs have been established at more than 40 or 50 years and risk has been modelled for each 10 years (OR 1.32, 95\% CI 1.07-1.63). A higher risk of lipoatrophy is also associated with male sex and lower fat mass before therapy ${ }^{28}$.

\section{Lipohypertrophy}

In the modern cART era, lipohypertrophy is generally observed in the abdomen and trunk ${ }^{48}$. Up to $70 \%$ of PLWH receiving cART have increased abdominal fat, which is now the most common type of fat alteration in these patients ${ }^{37,44,49,50}$, and risk factors include older age, female sex, elevated baseline triglycerides and higher baseline body fat percentage ${ }^{51}$. Generalized weight gain (see Generalized fat gain and obesity below) can be more difficult to distinguish from lipohypertrophy in persons with excess adiposity in the abdomen, chest, shoulder and nape of the neck (the android or 'apple' distribution) than those prone to accumulation in the hips, thighs and buttocks (the gynoid or 'pear' distribution). Mechanistically, lipohypertrophy is defined by regional increases in adipocyte size and/or number and it primarily affects the abdominal compartment, including disproportionate increases in VAT, dorso-cervical adipose tissue, breasts and supra-inguinal area, and diversely located lipomas ${ }^{28,52}$; of note, these changes are different to those observed with generalized fat gain.

Although lipoatrophy has been linked to specific ARTs, the independent risk of lipohypertrophy attributable to PI or NNRTIs use is difficult to assess as these agents were provided in combination with NRTIs ${ }^{48}$. Some studies of later-generation PIs have reported little difference in the size of anatomical areas prone to lipohypertrophy compared with NNRTIs or INSTIs ${ }^{51,53}$, with the possible exception of trunk fat gain with ritonavirboosted atazanavir ${ }^{54,55}$. Assessing disproportioned accumulation of adipose tissue, particularly in individuals with generalized weight gain and/or obesity, is aided by DXA and CT. Indeed, the imaging sub-study of the ACTG study A5257 demonstrated a mean CT-quantified VAT gain of $26 \% 96$ weeks after treatment initiation, with a $13 \%$ limb fat gain and $20 \%$ SAT gain, which did not vary significantly between individuals randomized to receive raltegravir, ritonavir-boosted darunavir or ritonavir-boosted atazanavir ${ }^{56}$. These findings suggest a disproportionate gain in VAT compared with SAT among persons receiving PIs or an INSTI in combination with newer-generation NRTIs. However, in a subsequent analysis, women had a greater increase in waist circumference on raltegravir versus PIs than men $^{57}$, suggesting sex differences in drug effects.

In long-term studies of ACTG participants, DXAquantified lean mass began to decline at 96 weeks after ART initiation, which could result in sarcopenia in the long term, relative to individuals without HIV, whereas trunk fat, associated with adverse metabolic outcomes, continued to accumulate at a faster rate than in individuals without $\mathrm{HIV}^{58}$. Similarly, in studies from the Modena HIV Metabolic Clinic, DXA-quantified trunk fat continuously and steadily increased, whilst lean mass consistently decreased, in PLWH using long-term cART, which was observed in both men and women and across all age groups ${ }^{59}$. As VAT gains of as little as $5 \%$ have been associated with an increased risk of metabolic syndrome ${ }^{60}$ and trunk fat accumulation has been associated with short-term mortality risk in $\mathrm{PLWH}^{61}$, patients with increased trunk adiposity have an increased risk of cardiometabolic diseases and could benefit from the screening and treatment of cardiovascular and metabolic risk factors.

\section{Generalized fat gain and obesity}

Weight and generalized fat gain are almost universally described following cART initiation, involve limb and trunk fat, SAT and VAT, and occur during the first year of treatment on all regimens ${ }^{8,9,56}$. In addition, the prevalence of obesity is increasing among $\mathrm{PLWH}$, in particular in North America and in sub-Saharan Africa ${ }^{62,63}$.

Weight gain following cART initiation could be attributable at least in part to a return to health phenomenon, with a concomitant gain in lean mass, but is clearly higher with some individual INSTIs, the newest antiretroviral class and TAF than with other ART molecules $^{12}$. In addition, weight gain is associated with personal factors, such as sex, genetics and ethnicity ${ }^{41,64}$. Thus, a greater fat gain both in the peripheral and truncal depots, resulting in generalized fat gain, has been observed in some individuals receiving INSTIs, both in cohort studies and in clinical trials ${ }^{10,41,65-68}$. Indeed, in a large analysis of more than 22,000 treatment-naive PLWH in the multi-site NA-ACCORD, individuals who initiated INSTI-based regimens gained an estimated mean $5.9 \mathrm{~kg}$ after 5 years of treatment, compared with $3.7 \mathrm{~kg}$ among individuals receiving NNRTI-based regimens and $5.5 \mathrm{~kg}$ for those receiving PI-based regimens ${ }^{69}$. Smaller observational studies have also reported a greater weight gain in PLWH initiating INSTI-containing regimens than in those initiating NNRTI or PI regimens, which generally has been greater in women, black individuals, and in those with lower weight and CD4 ${ }^{+}$ $\mathrm{T}$ cell counts before starting ART ${ }^{65,66,68,70}$. The aetiology of weight gain with INSTIs is unclear and may include effects on thermogenesis, appetite or energy regulation, or perhaps reflect a direct effect or a greater antiretroviral efficacy of these drugs in adipose tissue $e^{71,72}$ or other metabolically active tissue.

In the prospective, randomized ADVANCE study, an increased incidence of obesity was observed in ART-naive PLWH in South Africa who initiated dolutegravir than in those who initiated an efavirenz-based treatment ${ }^{11}$, affecting $7 \%$ of male patients and $20 \%$ of female patients initiating a dolutegravir-TAF-FTC regimen. Weight gain at 48 weeks was higher in women receiving dolutegravir-TAF-FTC (mean increase of $6.4 \mathrm{~kg}$ ) than in those initiating dolutegravir-TDFFTC (mean increase of $3.2 \mathrm{~kg}$ ) and women had a 
higher weight gain than men (mean increase of $4.7 \mathrm{~kg}$ for dolutegravir-TAF-FTC and $3.0 \mathrm{~kg}$ for dolutegravir-TDF-FTC); the reason for this sex difference is unknown ${ }^{11}$. In addition, a pooled analysis of weight gain in eight randomized controlled trials of treatment-naive PLWH initiating ART, comprising $>5,000$ participants, reported a higher weight gain at 96 weeks in those receiving TAF-containing regimens than in those receiving TDF, abacavir or zidovudine after adjusting for age, ethnicity, sex, baseline clinical factors and additional ART agents ${ }^{12}$.

Interestingly, the few studies of PLWH who switched from NNRTIs or PIs to INSTIs have reported mixed results. In a retrospective single-centre cohort study, there was no clear evidence of an overall increase in rate of weight gain following a switch to INSTIs in virologically suppressed individuals ${ }^{73}$. Differences in gender (mainly men) and ethnicity (mainly white) could partly explain this result. By contrast, an analysis of 691 patients with virologic suppression in the ACTG A5551 or A5332 studies from 2007 to 2017 found that women, black people and persons aged $\geq 60$ years experienced a greater rate of weight gain in the 2 years after a switch to an INSTI-based regimen than before the switch $^{74}$. Additionally, in the ANRS163 ETRAL study, 165 persons with viral suppression who switched from a PI to raltegravir and etravirine had a $12 \%$ increase in DXA-assessed total, trunk and limb fat but not in lean mass after 96 weeks ${ }^{75,76}$. The rate of weight gain is generally greater with dolutegravir than with elvitegravir or raltegravir, and among women, black people and those aged $\geq 60$ years $^{74,77}$.

\section{Ectopic fat depots}

Clinically apparent lipohypertrophy is one facet of a broader condition of ectopic lipid deposition in PLWH, which is also often observed in the liver, epicardial tissue and skeletal muscle. Indeed, several studies have demonstrated increased epicardial fat in cART-treated PLWH, the volume of which is associated with VAT area, insulin resistance, traditional risk factors for atherosclerosis and the duration of $\mathrm{cART}^{78-80}$. In addition, intramuscular fat accumulation has previously been reported in PLWH with lipoatrophy and/or lipohypertrophy ${ }^{81}$, and increased fatty infiltration of the thigh muscle was found to be associated with ageing independent of fat distribution $^{82}$. Increased hepatic fat content has also been observed in PLWH with lipoatrophy ${ }^{83}$ or in those with increased central adiposity ${ }^{84}$. Interestingly, and unique to PLWH, a parabolic association between SAT and liver fat has been observed, indicating high levels of steatosis in people with lipoatrophy and in those with increased subcutaneous adiposity ${ }^{83}$.

\section{Mechanisms/pathophysiology}

The mechanisms of fat alterations in PLWH are complex, multifactorial and only partly understood. The role of HIV itself is an area of increasing study, whereas the effect of ARTs has been proposed since the mid-1990s. Immune system activation, either locally or in the gut and with resulting inflammation, has also been proposed to affect adipocyte health.

\section{Adipose tissue as a reservoir for HIV}

Adipose tissue is an important HIV and simian immunodeficiency virus (SIV) reservoir, with the presence of latently infected $\mathrm{CD} 4^{+} \mathrm{T}$ cells and macrophages in both SAT and VAT depots from ART-naive PLWH and SIVinfected macaques ${ }^{85-87}$. Indeed, replication-competent $\mathrm{HIV}$ is present in both SAT and VAT CD4 ${ }^{+} \mathrm{T}$ cells from treated, aviraemic PLWH without clinical fat alterations, with a median latent HIV proviral DNA copy number equivalent to that in circulating and mesenteric lymph node $\mathrm{CD}^{+} \mathrm{T}$ cells. Additionally, SIV is detected within adipose tissue in ART-suppressed situations ${ }^{85-87}$ and HIV/SIV proteins can be detected in the plasma and tissues despite virus suppression ${ }^{88,89}$. Furthermore, SIV DNA and RNA are present in stromal vascular fraction cells, sorted $\mathrm{CD} 4^{+} \mathrm{T}$ cells and $\mathrm{CD} 14^{+}$macrophages in SAT and VAT from SIV-infected macaques ${ }^{85,87,90}$.

\section{Role of the virus}

As previously mentioned, many ART-naive PLWH have decreased overall fat mass and can have fat alterations affecting both SAT and VAT compartments, suggesting that HIV infection per se can affect adipose tissue and induce metabolic disturbances ${ }^{22,91,92}$ (FIG. 1).

ART-naive patients have a lower SAT volume and decreased whole tissue expression of CEPBA and PPARG (encoding the major transcription factors $\mathrm{C} / \mathrm{EBP} \alpha$ and PPAR $\gamma$, respectively, which are important for normal adipogenesis and cellular function) compared with HIV-negative controls ${ }^{93,94}$. Accordingly, the expression of pro-adipogenic genes is also reduced in SAT, including markers of lipid storage (LPL (encoding lipoprotein lipase) and FABP4 (encoding the fatty acid transporter 4)) and adipokines with important endocrine function (for example, LEP (encoding leptin) and $A D I P O Q$ (encoding adiponectin)), together with increased expression of proinflammatory cytokines such as IL-6 (REFS ${ }^{93,94}$ ). In accordance with altered adipose tissue function and fat loss in PLWH, adiponectin and leptin circulating levels are decreased in ART-naive PLWH ${ }^{95}$.

In addition, fat from both cART-treated and ART-naive PLWH has a decreased mitochondrial DNA (mtDNA) content and reduced expression of mitochondriaencoded proteins that are required for adequate adipocyte metabolic function ${ }^{93,94,96}$. Mitochondrial dysfunction is associated with increased reactive oxygen species (ROS) generation by the respiratory chain, which can impair adipocyte function and normal lipid storage and release $\mathrm{e}^{97}$. Interestingly, these effects are not dependent on circulating virus levels, as long-term HIV non-progressors not receiving cART (that is, PLWH with very low circulating viral levels and preserved $\mathrm{CD}^{+}$ $\mathrm{T}$ cell counts) demonstrate adipocyte mtDNA depletion and similar gene expression changes as observed in untreated PLWH with high viral levels ${ }^{94}$.

Morphological and functional analysis of adipose tissue from ART-naive macaques infected with SIV have revealed severe fibrosis in SAT and VAT characterized by collagen VI deposition and high expression of the profibrotic factor transforming growth factor- $\beta$ $(\mathrm{TGF} \beta)^{98}$. This fibrosis negatively correlates with adipocyte size and adipogenesis, suggesting that HIV infection 

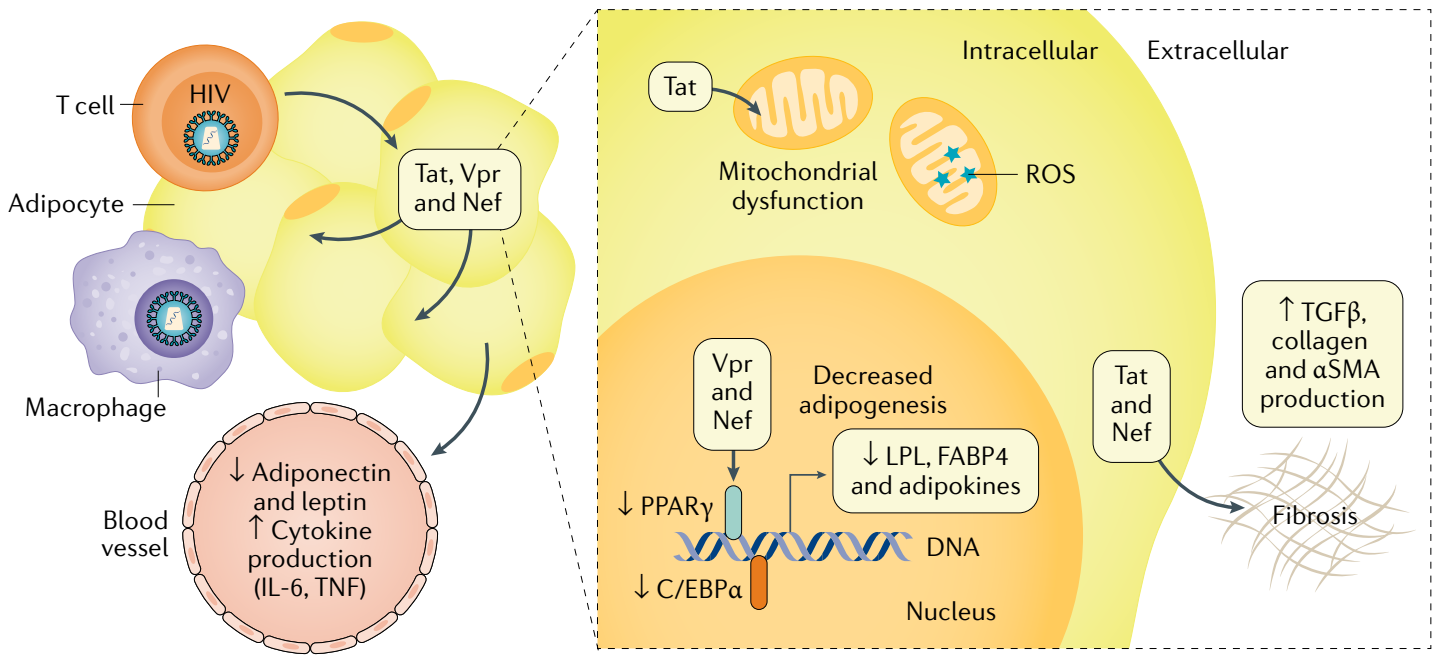

Fig. 1 | Effects of HIV on adipose tissue. HIV-infected T cells and macrophages can secrete viral proteins, such as Tat, Vpr and Nef, that affect proximal adipocytes, resulting in several cellular effects. These effects include mitochondrial dysfunction, increased production of extracellular matrix components leading to fibrosis, reduced adipogenesis, decreased production of adiponectin and leptin, and increased production of proinflammatory cytokines. $\alpha \mathrm{SMA}, \alpha$-smooth muscle

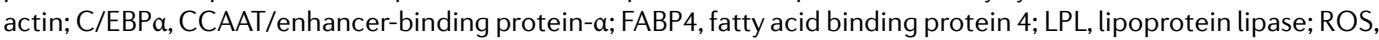
reactive oxygen species; PPAR $\gamma$, peroxisome proliferator-activated receptor- $\gamma$; TGF $\beta$, transforming growth factor- $\beta$; TNF, tumour necrosis factor.

per se could reduce adipose tissue expansion, at least in some depots. Although there are few data on adipose tissue fibrosis in treatment-naive PLWH, a longitudinal study in cART-treated PLWH with viral suppression found that adipose tissue fibrosis (assessed by changes in collagen VI and fibronectin deposition) decreased over 1 year in adults on cART with viral suppression ${ }^{99}$.

Latently infected $\mathrm{CD} 4^{+} \mathrm{T}$ cells and macrophages present within adipose tissue are thought to release viral proteins locally within adipose tissue and into the circulation with locally detectable levels, despite suppression of plasma viraemia by cART. The accessory proteins Vpr and Nef and the regulatory protein Tat are the main proteins secreted by infected cells and can exert bystander effects on neighbouring adipocytes or precursor cells and affect adipose tissue metabolism ${ }^{100,101}$ (FIG. 2). In vitro studies have been used to evaluate how viral proteins affect adipocyte function, although it should be noted that these studies generally used viral protein concentrations observed in ART-naive patients but which could correspond to concentrations in adipose tissue. In these studies, Vpr and Nef have been shown to suppress the activity of the adipocyte master regulatory protein PPAR $\gamma$, leading to decreased expression of genes involved in the regulation of lipid storage (for example, LPL and HSL (encoding hormone sensitive lipase) and FABP4 and CD36, involved in fatty acid transport $)^{90,98,102-104}$. The role of Tat is less clear than that of Vpr and Nef, as it can inhibit or promote adipogenesis depending on the cellular model used ${ }^{98,105,106}$. In some studies, Tat has been shown to induce mitochondrial dysfunction, which likely occurs through mitochondrial membrane permeabilization and generation of mitochondrial ROS ${ }^{107}$. In addition, Tat and Nef have been shown to promote the acquisition of a profibrotic phenotype characterized by the expression of TGF $\beta$ and $\alpha$-smooth muscle actin ( $\alpha$ SMA) and an increased production of extracellular matrix components such as collagen I, VI and fibronectin, leading to increased fibrosis, which corresponds with the in vivo data from macaques and cART-treated $\mathrm{PLWH}^{98}$. Collectively, these data indicate that HIV infection can directly affect adipose tissue function and morphology through the action of viral proteins.

\section{Role of adipose immune system}

Adipose tissue contains cells of the innate and adaptive immune systems that defend against a range of pathogens, aid in the removal of apoptotic adipocytes and other cellular debris, and affect adipocyte regulation as well as energy storage and release. Research into obesity has established both the malleability and the importance of the adipose tissue immune environment for metabolic health and serves as a context to interpret adipose tissue changes in PLWH (FIG. 3). Obesity is accompanied by progressive accumulation of $\mathrm{CD}^{+} \mathrm{T}$ cells, $\mathrm{CD} 4^{+}$ $\mathrm{T}$ helper $1\left(\mathrm{~T}_{\mathrm{H}} 1\right)$ and $\mathrm{T}_{\mathrm{H}} 17$ cells, and M1-phenotype proinflammatory macrophages (which are $\mathrm{CD}^{+} 8^{+}$and produce tumour necrosis factor (TNF), IL-12 and IL-23) in adipose tissue, with concomitant reduction of $\mathrm{T}_{\mathrm{H}} 2$ and regulatory $\mathrm{T}$ cells ${ }^{108-111}$. The mechanistic importance of these immune changes is demonstrated by animal studies showing that experimental depletion of adipose tissue $\mathrm{CD}^{+} \mathrm{T}$ cells improves tissue inflammation and insulin resistance in obesity ${ }^{110}$.

Early studies of subcutaneous fat from PLWH receiving cART with lipoatrophy reported greater adipocyte apoptosis, fibrosis and expression of the inflammatory markers IL-12, p40, IL-6, IL-8 and macrophage inflammatory protein $1 \alpha$ (MIP1 1 ) than in tissue from individuals without HIV, which was thought to be principally due to the effects of NRTIs on adipocyte function ${ }^{112}$. 
High levels of these cytokines inhibit adipocyte insulin signalling by reducing gene expression of IRS1, phosphoinositide 3-kinase p85a and GLUT4 (REFS ${ }^{113-118}$ ). Despite the increased inflammatory and macrophage activation markers (for example, MIP1 $\alpha$ ) in adipose tissue from PLWH, findings on adipose tissue macrophage density have been varied ${ }^{112,118}$. One study reported greater apoptosis, fibrosis and macrophage infiltration, reduced expression of the genes encoding adiponectin and leptin, and higher IL- 6 and TNF in PLWH with peripheral lipoatrophy using a PI-based cART regimen compared with people without $\mathrm{HIV}^{118}$. By contrast, another large study found that macrophage density did not differ between PLWH and individuals without HIV or by lipoatrophy status in PLWH, although macrophage density was slightly higher in individuals receiving zidovudine and stavudine than in those receiving abacavir and tenofovir ${ }^{112}$. In this study, PLWH with clinically evident lipoatrophy had higher HIV-DNA levels within circulating $\mathrm{CD} 14^{+} \mathrm{CD} 16^{+}$monocytes than in PLWH without lipoatrophy, but the mechanisms linking this monocyte viral reservoir with fat alteration have yet to be elucidated ${ }^{112}$.

In the current cART era, several studies have reported a profound shift in the adipose tissue $\mathrm{T}$ cell profile towards a $\mathrm{CD}^{+}$cell predominance in both PLWH and macaques with SIV infection ${ }^{86,119,120}$, which is intriguing given the $\mathrm{CD} 8^{+} \mathrm{T}$ cell infiltration in obesity and the higher rates of metabolic disease in $\mathrm{PLWH}^{46,121-123}$. Interestingly, among PLWH seen in a European metabolic diseases clinic with a range of fat alteration phenotypes, circulating $\mathrm{CD}^{+} \mathrm{T}$ cell activation was higher in both patients with lipoatrophy and in those with central fat accumulation than in patients without clinically apparent fat redistribution ${ }^{124}$. In addition, $\mathrm{CD}^{+} \mathrm{T}$ cells in SAT from PLWH without clinically apparent lipodystrophy have increased antigen receptor clonality ${ }^{120}$, a finding which is also reported in animal models of obesity ${ }^{125}$. This finding could reflect local $\mathrm{T}$ cell expansion, although some studies have reported a lack of Ki-67 (a marker of proliferating cells) expression and high CD57 (a marker of late differentiation or senescence and an indicator of reduced replicative capacity) expression on $\mathrm{CD}^{+}$cells from adipose tissue of SIV-infected macaques, which could reflect minimal in situ proliferation ${ }^{87,126,127}$. Interestingly, the distribution of $\mathrm{CD}^{+}$and $\mathrm{CD} 8^{+} \mathrm{T}$ cell subsets (naive, central memory, effector memory and effector memory $\mathrm{RA}^{+} \mathrm{T}$ cells) was similar in SAT from PLWH without clinically evident lipodystrophy, suggesting that the accumulation of $\mathrm{CD}^{+} \mathrm{T}$ cells occurs in a relatively stochastic manner, as opposed to accumulation of one broad subset ${ }^{128}$. Furthermore, the same study found the SAT of PLWH with T2DM to be markedly enriched for CD4 ${ }^{+} \mathrm{T}$ effector memory cells and $\mathrm{T}$ effector memory RA cells co-expressing CD57, CX $\mathrm{CR}_{3} 1$ and GPR56 compared with the SAT of non-diabetic PLWH, a phenotype which is reported to have antiviral specificity ${ }^{129-132}$.

\section{Role of ART}

Several studies have examined SAT morphology and function in PLWH and lipodystrophy receiving cART (FIC. 2a). The main features in lipoatrophy-prone SAT were a markedly decreased gene and protein expression of pro-adipogenic transcription factors, including PPAR $\gamma, \mathrm{C} / \mathrm{EBP} \alpha$ and SREBP1, and of their target genes, such as GLUT4 or LPL, compared with HIV-negative controls (although the molecular mechanism of this is unknown), leading to decreased adipocyte differentiation and resulting in impaired fatty acid and glucose metabolism ${ }^{23,133}$. Furthermore, probably due to impaired adipogenesis, the expression of adiponectin and leptin (markers of mature adipocytes) was reduced ${ }^{100,133}$. In addition, chronic low-grade inflammation has been observed in lipoatrophic SAT from PLWH, characterized by increased gene and protein expression of proinflammatory markers (such as IL-6 and TNF). These proinflammatory markers are secreted by infiltrating immune cells and stressed adipocytes and are associated with increased fibrosis, TGF $\beta$ expression and increased adipocyte apoptosis ${ }^{118,134-136}$. SAT loss owing to thymidine NRTIs is accompanied by a shift to a proinflammatory and profibrotic state, which does not fully resolve following the removal of these agents ${ }^{48}$.

Loss of limb fat was more prevalent with older thymidine analogues (for example, zidovudine or stavudine) than with newer NRTIs (for example, lamivudine, abacavir and tenofovir), and there is evidence that the mtDNA haplogroup may influence susceptibility ${ }^{9,137-143}$. Older thymidine analogue NRTIs seem to inhibit mtDNA polymerase- $\gamma$ most strongly, resulting in reduced mtDNA replication and content per cell ${ }^{144,145}$, including substantial reductions in the mtDNA content of adipocytes ${ }^{138,146,147}$. Reduced mtDNA levels are probably responsible for the reduced metabolic flexibility of lipoatrophic adipose tissue ${ }^{138,148}$. The mtDNA haplogroup may influence susceptibility to NRTI-induced lipoatrophy ${ }^{142,146,149}$, with mitochondrial haplogroups $\mathrm{H}$, $\mathrm{I}$ and $\mathrm{K}$ conferring a greater risk of lipoatrophy and haplogroups $\mathrm{T}$ and $\mathrm{W}$ being protective against lipoatrophy ${ }^{140,141,150}$. Variation in mtDNA among haplogroups is speculated to influence energy production efficiency, ROS generation and adipocyte apoptosis. Haplogroup $\mathrm{H}$, in particular, is believed to be tightly coupled to ATP production and, consequently, to more ROS generation, whereas haplogroup $\mathrm{T}$ is partially uncoupled to ATP production, leading to less ROS production ${ }^{140,141,150}$. Oxidative stress has also been proposed to underlie thymidine analogue NRTI-related mitochondrial toxicity by inducing mtDNA depletion and subsequent mitochondrial energy deficiency, leading to adipocyte loss and tissue atrophy ${ }^{150}$.

In comparison to SAT, only a few studies have evaluated the alterations of VAT in ART-treated PLWH. A study comparing PLWH with lipoatrophy and central fat accumulation with healthy controls found similar mtDNA depletion and abnormal increases in mitochondrial protein levels in VAT and SAT from PLWH, whilst transcript levels of adipogenesis marker genes, such as $P P A R G$ and $A D I P O Q$, were unaltered in VAT but were decreased in SAT ${ }^{151}$. Moreover, SAT from PLWH with lipoatrophy had increased glucose uptake, possibly a compensatory mechanism for reduced stores on PET compared with healthy controls, whereas VAT glucose uptake was the same irrespective of HIV status ${ }^{152}$. 


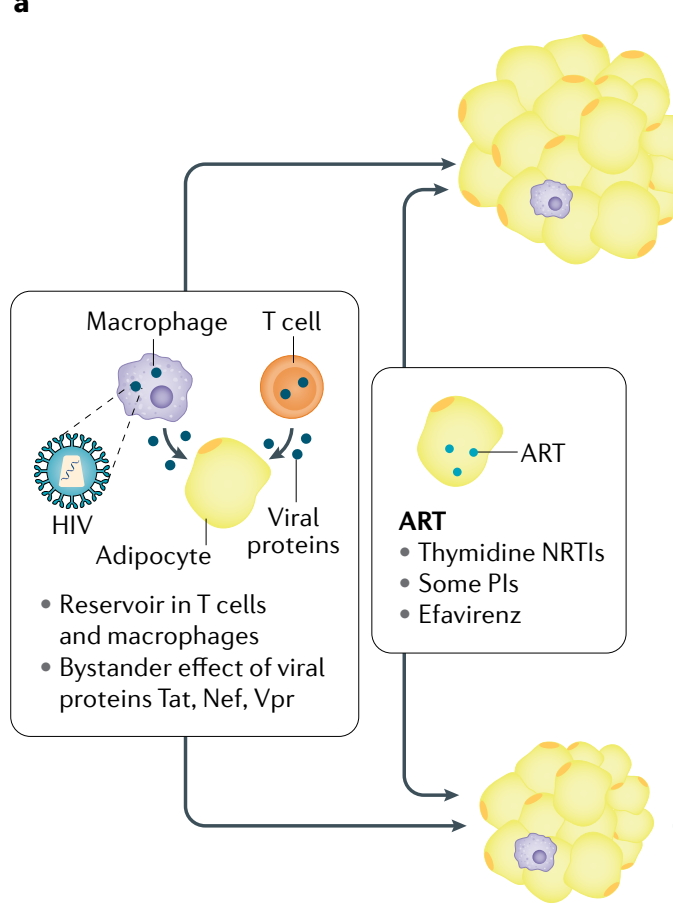

SAT deficiency

-+++ Mitochondrial dysfunction

++ Inflammation

-++ Fibrosis

$\bullet++$ Adipogenesis alteration

b

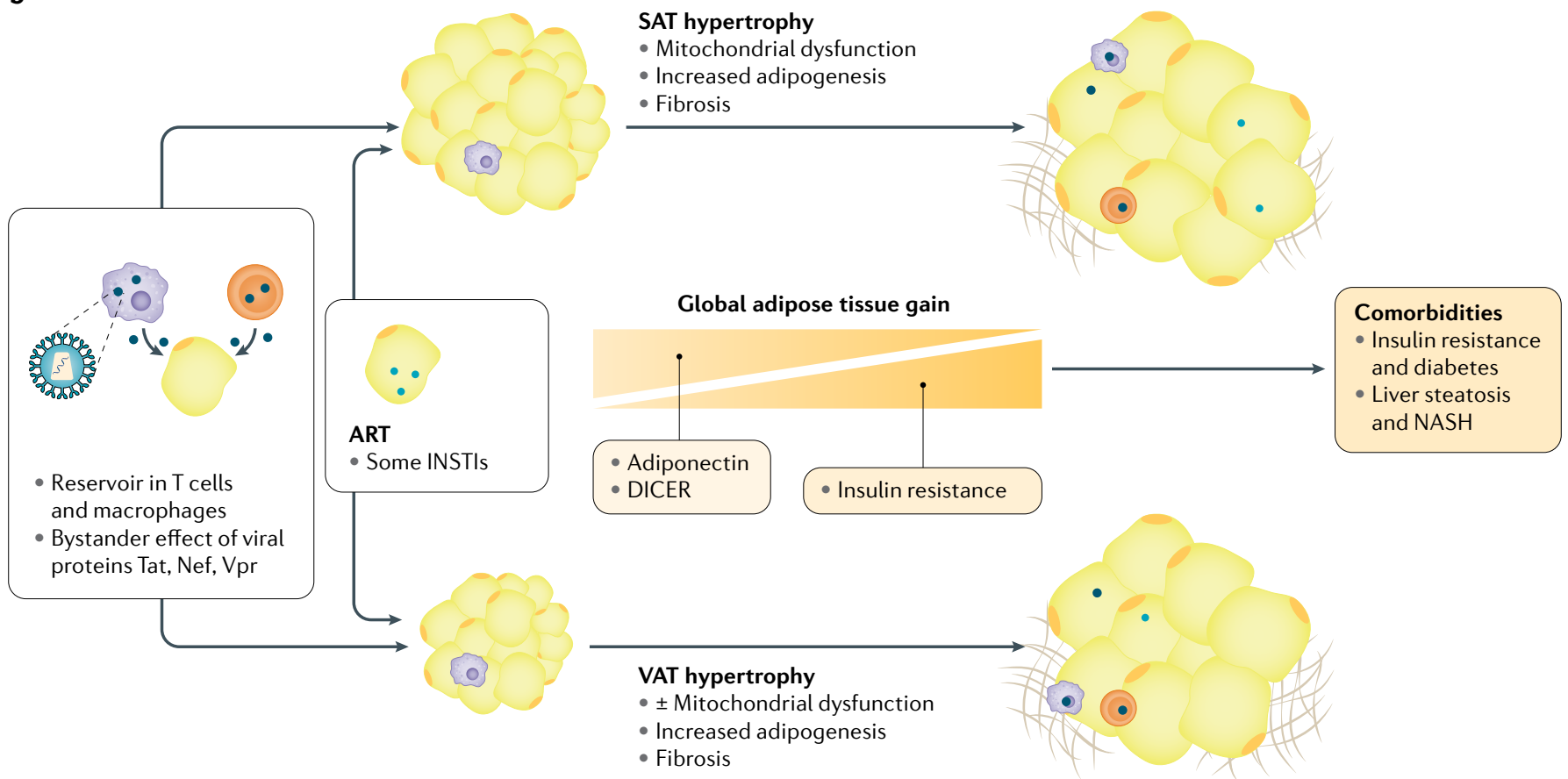

Thus, SAT but not VAT from lipodystrophic PLWH has an increased metabolic rate (that is, higher rates of glucose transport and lipid oxidation), suggesting a greater metabolic flexibility for SAT and a capacity for expansion for VAT ${ }^{152-154}$. Molecular analysis of SAT from PLWH and lipoatrophy found reduced expression of the microRNA-processing enzyme DICER, which is associated with the downregulation of brown and beige adipose tissue genes implicated in mitochondrial biogenesis such as UCP1 and PPARGC1 $A^{154,155}$. This phenotypic change could, in part, explain cART-related increased oxidative stress and mitochondrial toxicity, which could also contribute to the metabolic dysregulation observed in adipose tissue of PLWH. DICER expression was strongly and negatively associated with the duration of PI use ${ }^{156}$ (FIG. 2). In addition, the duration of cART and PI use was linked with the reduced expression of brown and beige fat genes that are involved 
4 Fig. 2 | Pathophysiological mechanisms involved in HIV-related and ART-related adipose tissue alterations and dysfunction. Subcutaneous adipose tissue (SAT) and visceral adipose tissue (VAT) alterations are often observed among people living with HIV. Some insults to adipose tissue could result from HIV infection. HIV is present in certain T cells and macrophages within adipose tissue. HIV proteins, such as Tat, Nef and Vpr, released by HIV-infected immune cells within adipose tissue, can exert bystander effects on proximal adipose cells. a $\mid$ Some alterations can be related to individual antiretroviral therapy (ART) molecules among the different classes, in particular thymidine nucleoside reverse transcriptase inhibitors (NRTIs), some protease inhibitors (Pls) and non-NRTIs (NNRTIs) such as efavirenz. The development of a profibrotic, proinflammatory and anti-adipogenic environment is more likely in SAT than in VAT. In some cases, adipocyte death occurs with surrounding macrophages. Adipocyte stress and dysfunction as a result of mitochondrial dysfunction, decreased adiponectin and DICER expression, and increased release of proinflammatory cytokines such as IL- 6 and tumour necrosis factor (TNF) by adipocytes and immune cells could lead to adipocyte insulin resistance and storage deficiency, ultimately driving lipid overflow. Thus, SAT deficiency, combined with VAT hypertrophy, may convey lipotoxicity, lipid accumulation in the liver, heart, vessels, muscles and pancreas, and related comorbidities such as type 2 diabetes mellitus, liver steatosis and non-alcoholic steatohepatitis (NASH), and atherosclerotic cardiovascular diseases. $\mathbf{b} \mid$ Alterations at the adipose tissue level induced by some integrase strand transfer inhibitors (INSTIs) appear partly different. These molecules can induce adipocyte hypertrophy with increased adipogenesis together with increased fibrosis and mitochondrial dysfunction, which could result in global fat gain with increased SAT and VAT depots as well as in increased peripheral fat. Insulin resistance and liver steatosis are expected outcomes. +++, a major effect; ++, an important effect; +, a mild effect; \pm , the effect is minor or absent.

in thermogenesis, which could mediate metabolic complications ${ }^{156}$. Finally, rodent models have shown that thymidine analogue exposure induces greater mtDNA depletion in SAT than in VAT, whereas in vitro exposure of adipose tissue to older generation PIs increases lipolysis and decreases glyceroneogenesis (a metabolic pathway that recycles free fatty acids to triglycerides) in SAT but not in VAT ${ }^{153,157}$.

As previously mentioned, some PLWH have hypertrophy of dorsocervical adipose tissue (DCAT; referred to as a 'buffalo hump'), with a prevalence of up to $25 \%$ in PLWH in the $1990 \mathrm{~s}^{158}$. Interestingly, DICER expression in SAT is inversely associated with excess DCAT accumulation $^{149}$. Similar to PLWH with clinical fat alteration, DICER adipocyte-specific knockout mice have reduced white adipose tissue depots (lipoatrophy), whitening of brown adipose tissue depots, insulin resistance and local inflammation ${ }^{159,160}$. However, although hypertrophied DCAT from PLWH has increased fibrosis gene expression, studies show less inflammation and less disruption of adipogenesis than expected from hypertrophied depots ${ }^{161}$, suggesting that DCAT has different characteristics to other fat depots ${ }^{162}$. The downregulation of DICER in SAT of PLWH may favour a shift to a proinflammatory and profibrotic state, limiting the capacity for fat browning and storage. Taken as a whole, lipohypertrophy, including the enlargement of DCAT, in PLWH has been hypothesized to result, in part, from impaired energy storage in atrophied, inflamed and fibrotic SAT ${ }^{100,163}$.

A number of in vitro studies have been conducted to investigate the individual effect of ART on adipocytes, although results from these studies need confirmation with clinical data in PLWH. These studies revealed effects of PIs and NRTIs on adipogenesis, insulin signalling, adipokine secretion and adipocyte apoptosis ${ }^{23,163}$. As previously mentioned, thymidine analogue NRTIs induce mitochondrial toxicity, in part, by inhibiting the mtDNA polymerase- $\gamma^{138,146,149}$. The effect of PIs on adipose tissue could result from the inhibition of ZMPSTE24 (which cleaves prelamin A to lamin A), which could lead to defects in lamin A maturation and organization and, consequently, to SREBP1 nuclear mislocalization and reduced adipogenesis ${ }^{164}$. Moreover, ZMPSTE24 inhibition leads to the accumulation of senescence-associated prelamin $\mathrm{A}$, also observed in circulating immune cells and in adipose tissue of $\mathrm{PLWH}^{161,165,166}$, causing cellular senescence and adipocyte dysfunction. PIs could also lead to enhanced endoplasmic reticulum stress owing to high ROS production, which could also have adverse effects on adipocyte function ${ }^{23}$. Many of these cellular alterations are absent or less severe with modern PIs (such as atazanavir or darunavir) ${ }^{167,168}$. Among NNRTIs, efavirenz can affect in vitro adipogenesis and ADIPOQ expression whilst increasing the expression of proinflammatory markers ${ }^{169}$.

Early in vitro studies suggested that the INSTIs elvitegravir and raltegravir do not alter adipogenesis of cultured adipocytes ${ }^{170-172}$; however, more recent studies of SAT and VAT from INSTI-treated macaques displayed increased fibrosis, adipocyte size and adipogenic marker expression (PPARG and CEBPA) compared with untreated animals. Similarly, SAT and VAT from INSTI-treated PLWH had a higher level of fibrosis than SAT and VAT from non-INSTI-treated patients. Data from in vitro studies support the biopsy data, as dolutegravir and, to a lesser extent, raltegravir increased extracellular matrix production and lipid accumulation in human adipose stem cells and/or adipocytes ${ }^{72}$. Despite their pro-adipogenic effect, INSTIs have also been shown to promote oxidative stress and insulin resistance $^{72}$ (FIG. 2b).

Changes in gene expression, such as reduced expression of brown and beige fat genes (UCP1, PPARGC1A and PRDM16) ${ }^{156}$, has also been reported in SAT from INSTI-treated PLWH and could favour adipocyte hypertrophy. In addition, SAT from PLWH who have been switched to raltegravir/maraviroc exhibited decreased expression of gene pathways linked to immune function and activation ${ }^{173}$. One hypothesis would be that, in PLWH, INSTIs could alleviate adipocyte stress by promoting hypertrophy and expansion, resulting in fat gain, but may also lead to increased oxidative stress, fibrosis and insulin resistance.

INSTIs can also have direct or indirect effects on food intake through effects on MC4R in the hypothalamus $^{10}$, on thermogenesis and energy regulation ${ }^{156}$, and on the hormonal milieu (as indicated by the protective level of the anti-Müllerian hormone against raltegravir-induced fat gain in women with reproductive activity ${ }^{76}$. Moreover, the notable heterogeneity of weight gain in PLWH who received INSTIs raises the possibility of genetic susceptibility in energy signalling or storage pathways. Further clinical studies are needed to explore the effects of INSTIs on adipose tissue health and globally in vivo.

Adipose tissue can impair the efficacy of cART, which can contribute to viral persistence. Indeed, adipocytes can sequester substantial amounts of ARTs inside 
a Lean, HIV-negative fat

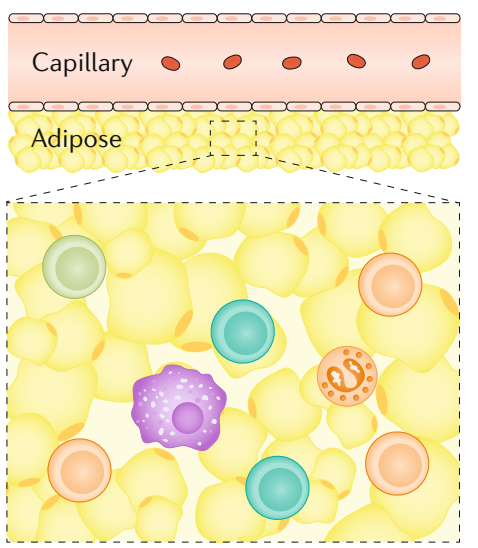

b Obese, HIV-negative fat

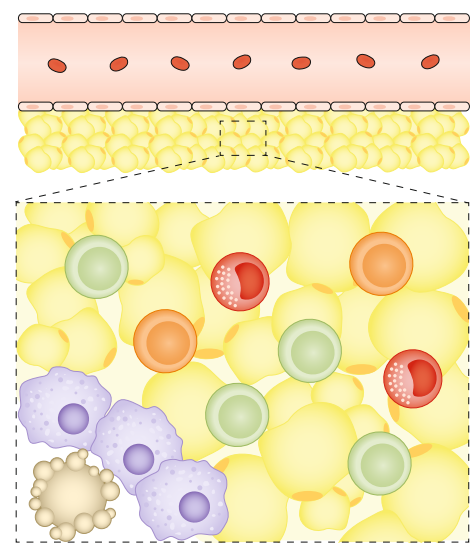

C HIV-positive fat

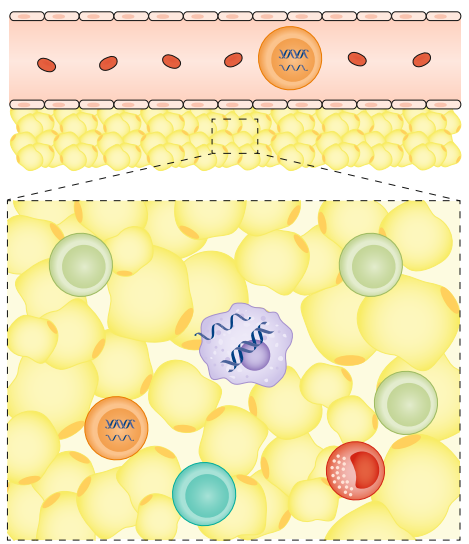

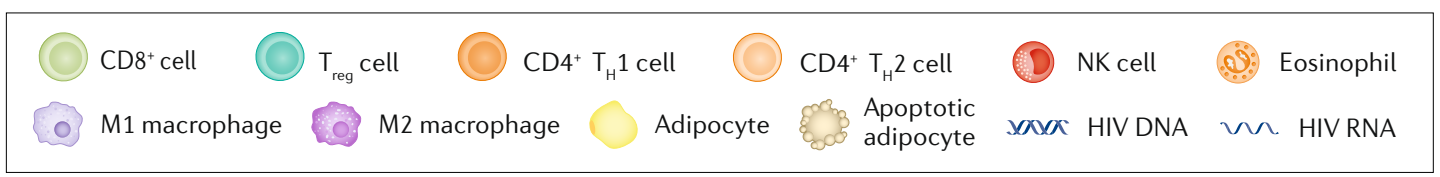

Fig. 3 | Alterations of immunity in adipose tissue from HIV-infected individuals with fat redistribution as compared with lean and obese non-infected individuals. a | Adipose tissue from lean individuals is comprised of adipocytes and a stromal vascular cell fraction in which $C D 4^{+} T$ cells predominate over $C D 8^{+} T$ cells and are typically polarized towards a T helper $2\left(\mathrm{~T}_{\mathrm{H}} 2\right)$ cell cytokine profile. The adipose tissue also contains macrophages characterized by an anti-inflammatory (M2) phenotype, regulatory $T\left(\mathrm{~T}_{\text {reg }}\right)$ cells and eosinophils with few natural killer (NK) cells. b $\mid$ Obese adipose tissue is characterized by hypertrophic and more heterogeneous adipocytes, an increased proportion of $\mathrm{CD} 8^{+} \mathrm{T}$ cells with more oligoclonal $\mathrm{T}$ cell receptor repertoires, $\mathrm{CD} 4^{+} \mathrm{T}$ cells polarized towards a $\mathrm{T}_{\mathrm{H}} 1$ cell cytokine profile and fewer $\mathrm{T}_{\text {reg }}$ cells. In animal models, the influx of $\mathrm{CD} 8^{+} \mathrm{T}$ cells in obesity promotes recruitment and activation of macrophages with a proinflammatory (M1) phenotype, some of which surround apoptotic adipocytes. There are fewer eosinophils and more NK cells. c | Adipose tissue from (non-obese) persons with HIV, irrespective of the presence of clinically apparent lipodystrophy, demonstrates several similarities but also notable differences with obesity. In HIV and in simian immunodeficiency-infected macaques, adipocytes are similar in size to those in lean, HIV-negative persons but can demonstrate greater heterogeneity and impaired adipogenesis and lipid metabolism. There is a marked enrichment in $C D 8^{+} T$ cells over $C D 4^{+} T$ cells but few infiltrating macrophages. The $C D 4^{+} T$ cells are predominantly $C D 69^{+}$, the $\mathrm{T}_{H} 1 / T_{H} 2$ cell balance is similar to that in lean, HIV-negative persons, and the number of $\mathrm{T}_{\text {reg }}$ cells may be similar or increased. In HIV and SIV, a notable exception is the presence of latently HIV/SIV-infected CD4 ${ }^{+}$T cells capable of producing viral proteins and RNA virus.

the adipocyte lipid droplet, which renders the therapy ineffective ${ }^{174}$. Adipocytes have consistently been shown to reduce the antiviral efficacy of TDF and TAF in vitro and analysis of adipose tissue from treated PLWH suggests that INSTIs penetrate adipose tissue, whereas the penetration of NRTIs is restricted ${ }^{71}$. Obesity has been reported to affect ART plasma exposure but without consequence on immunovirological control ${ }^{175}$.

\section{Role of gut dysbiosis and inflammation}

Gastrointestinal dysbiosis (a microbial imbalance or maladaptation characterized by underrepresentation of typically dominating species and overrepresentation of normally outcompeted or contained species) is emerging as an important contributor to adipose tissue dysfunction in PLWH. Alterations in the human gut microbiome have been linked to increased risk of obesity $^{176}$, cardiovascular disease ${ }^{177}$, hypertension ${ }^{178}$ and $\mathrm{T}^{2} \mathrm{DM}^{179,180}$, among other conditions.

HIV infection alters the architecture of the intestinal wall and the composition of the gut microbiome in PLWH, which persists despite effective viral suppression using cART and has detrimental effects on mucosal immunity, systemic inflammation and metabolic function ${ }^{181,182}$.
The intestinal mucosa undergoes villous atrophy, increased enterocyte apoptosis, impairment of tight junctions, impaired $\mathrm{B}$ cell function, decreased IgA production and depletion of T cells (especially $\mathrm{T}_{\mathrm{H}} 17$ cells) ${ }^{183}$, which can occur as soon as 2 weeks after infection in SIV-infected macaques ${ }^{184}$. During the acute infection phase, HIV replication depletes $80-90 \%$ of gut $\mathrm{CD} 4^{+} \mathrm{T}$ cells, which promotes mucosal inflammation, affects the immune response to translocating bacteria and leads to increased activated circulating $\mathrm{CD}^{+} \mathrm{T}$ cells ${ }^{185-189}$. In addition, microbial dysbiosis, predominantly characterized by reduced diversity within the microbiota (similar to the findings in individuals with obesity), is evident before the onset of marked immunosuppression in HIV infection ${ }^{187}$ and persists in chronic infection ${ }^{190-198}$. Specifically, members of the Proteobacteria phyla in the Enterobacteriaceae family, including Salmonella, Escherichia, Shigella, Klebsiella and Serratia species, are enriched in viraemic, untreated PLWH compared with HIV-negative individuals, whereas Bacteroidetes and Firmicutes are reduced ${ }^{199}$, although findings have varied between studies ${ }^{195,200,201}$. cART initiation does not seem to restore the microbiota to a pre-infection community composition ${ }^{202}$ and could actually further reduce diversity ${ }^{192,196}$. 
The translocation of microbial products promotes VAT inflammation ${ }^{203}$, impairs adipocyte energy handling and has been shown to contribute to the accumulation of visceral fat in the general population ${ }^{203}$; this mechanism has also been suggested in $\mathrm{PLWH}^{204}$. Indeed, the translocation of lipopolysaccharide (LPS) across the gut wall promotes adipose tissue inflammation $^{203}$ owing to the activation of adipose tissue macrophages via the $\mathrm{CD} 14$ receptor, causing a shift from an M2 to an M1 phenotype, and increases IL-6, TNF and other inflammatory cytokine production ${ }^{203}$. Circulating and locally produced VAT TNF promotes further loss of tight junction integrity and gut barrier dysfunction in a cyclic fashion ${ }^{205}$. Indeed, in ART-initiated PLWH from the ACTG A5257/A5260s study, gut integrity was associated with VAT gain and higher baseline intestinal fatty acid-binding protein levels were associated with increases in VAT, total body fat and BMI after 96 weeks $^{204}$.

A potential role for circulating LPS endotoxaemia in adipose tissue expansion is evidenced by animal studies demonstrating that a 4-week LPS infusion increases body weight and VAT to the same extent as a 4-week high-fat diet regimen and increases insulin resistance and expression of inflammatory transcripts in adipose tissue $\mathrm{e}^{203}$. LPS stimulates the proliferation of pre-adipocytes through a CD14-dependent mechanism ${ }^{205}$, possibly through activation of JAK/ STAT and AMPK via cytosolic phospholipase A2 (REF. ${ }^{206}$ ).
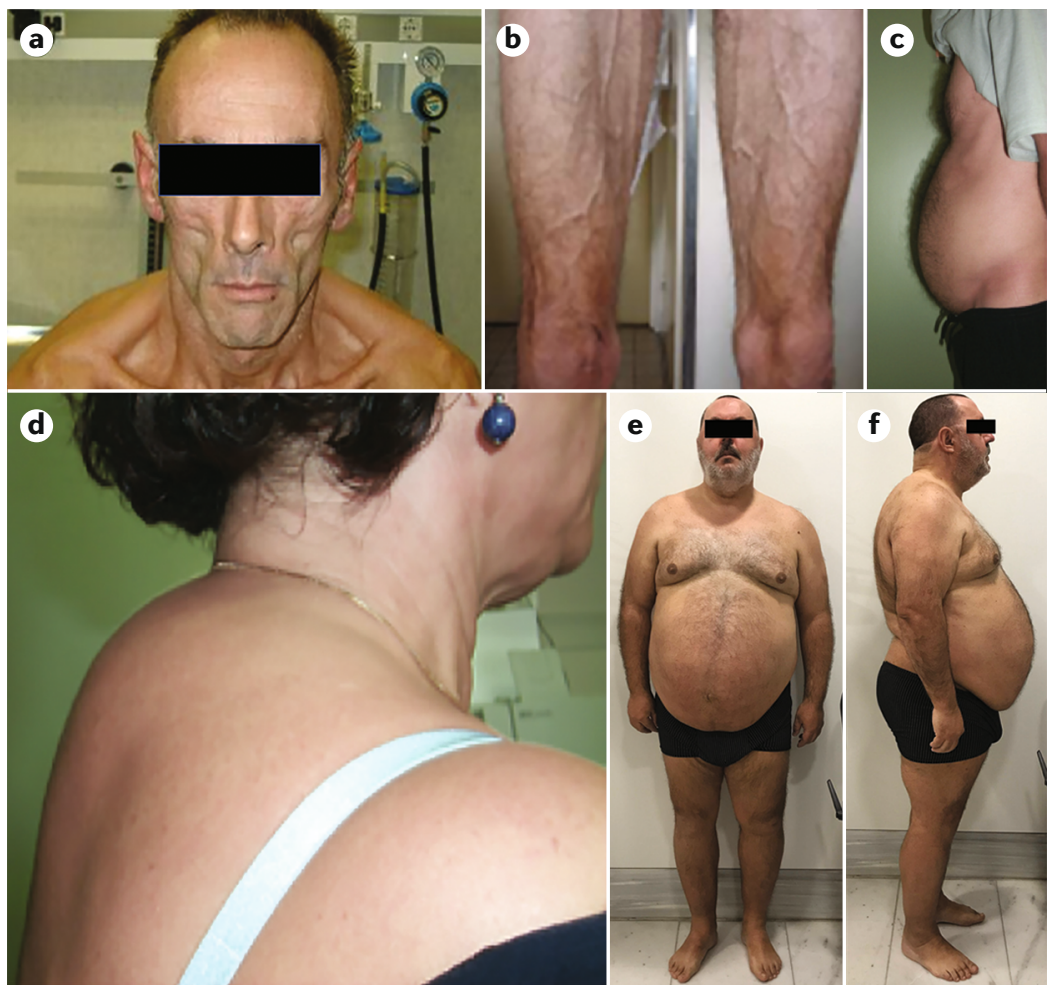

Fig. 4 | Manifestations of ART-associated fat alterations. Facial lipoatrophy related to thymidine nucleoside reverse transcriptase inhibitors (part a). Limb lipoatrophy related to thymidine nucleoside reverse transcriptase inhibitors (part b). Abdominal lipohypertrophy (part c). Buffalo hump (part d). Fat gain leading to obesity in a patient treated with an integrase strand transfer inhibitor (parts $\mathbf{e}$ and f). ART, antiretroviral therapy.
In addition, higher levels of LPS absorbed via chylomicrons among persons on high-fat diets is hypothesized to increase adipocyte lipid uptake in obesity ${ }^{206}$, although a similar link between LPS inclusion in lipid droplets and adipocyte hypertrophy in the context of HIV-related dysbiosis and gut barrier dysfunction requires further investigation. It should be noted that, although gut dysbiosis and impaired mucosal barrier function have been observed in PLWH, the mechanistic linkages and the directionality of this association are not fully understood $^{195,207}$.

\section{Diagnosis, screening and prevention \\ Diagnosis and clinical assessment}

The diagnosis of fat alterations in PLWH is primarily based on clinical assessment, patient medical history and patient self-report ${ }^{208}$. Indeed, both regional and generalized accumulation or loss of fat mass beyond what is expected for age-related changes in body composition according to local standards is sufficient for diagnosis (FIG. 4). Diagnosis of lipoatrophy or lipohypertrophy may lead to a need for screening for additional health problems, such as T2DM and dyslipidaemia, owing to the well-described associations between these metabolic disturbances and the presence of lipoatrophy and/or lipohypertrophy.

In general, the diagnosis of lipoatrophy, particularly of the face and periphery, is performed via visual inspection. Skinfold thickness measurement can be used for research purposes but is rarely used in clinic practice owing to its poor additional clinical usefulness.

Lipohypertrophy specifically refers to localized abnormal fat accumulation on physical examination or imaging, most frequently of the VAT, breasts, DCAT and/or as discrete accumulations under the skin (lipomas). Lipohypertrophy can occur in one or more regions simultaneously, with or without concurrent peripheral lipoatrophy. Importantly, the clinical assessment of lipohypertrophy can be complicated by concomitant obesity in PLWH. Obesity is traditionally defined as a BMI $>30 \mathrm{~kg} / \mathrm{m}^{2}$; however, low muscle mass and increased VAT to BMI ratios ${ }^{209-211}$, both of which are common in PLWH, can lead to underestimations of regional and generalized adiposity. A personal history or family history of obesity and diffuse fat distribution can support the diagnosis of generalized obesity in PLWH, whereas the diagnosis of lipohypertrophy can be supported by regional truncal or visceral fat accumulation. Of note, lipohypertrophy is typically clinically visible at least 1-2 years after the commencement of cART ${ }^{212}$. Although most standardized lipohypertrophy definitions include radiographic assessment of fat quantity ${ }^{4}$, the use of imaging is generally restricted to research settings $^{213-215}$. In addition, cohorts used to generate the definitions of lipohypertrophy were predominantly comprised of PLWH who received early-generation NRTIs and, therefore, had a high prevalence of lipoatrophy with concomitant lipohypertrophy ${ }^{216}$, meaning that the validity of these definitions in the present ART era is unknown. Of note, the gain of fat reported with INSTI use is generalized in most patients and is not defined as lipohypertrophy. 
In clinical practice, body weight and BMI should be tracked at least once per year in PLWH. In addition, as BMI can underestimate adiposity in PLWH, measurement of waist circumference is also recommended at least annually ${ }^{217,218}$. A minimum waist circumference of $\geq 94 \mathrm{~cm}$ in women and $\geq 95 \mathrm{~cm}$ in men correlates with a VAT area of $\geq 130 \mathrm{~cm}^{2}$, which is a validated threshold for increased cardiometabolic risk ${ }^{219,220}$, although these values have not been validated in PLWH. The causes of lipohypertrophy are not fully understood and are likely multifactorial. Generalized obesity and central lipohypertrophy can overlap in an ageing population with traditional and HIV/ART-specific risk factors, which can confound the diagnosis of lipohypertrophy.

\section{Prevention}

HIV seeding into fat is believed to occur early in HIV infection, and initiation of cART during acute HIV infection, which has been shown to decrease other HIV tissue reservoirs ${ }^{221,222}$, could limit HIV-induced adipose tissue dysfunction. In addition, the use of ARTs with better adipose tissue penetration, such as INSTIs, might also help reduce reservoir size and, potentially, HIV-associated fat depot disturbances. However, the use of INSTIs should be balanced against evidence that some INSTIs increase fat mass ${ }^{11,59}$. Earlier cART initiation also avoids progression of HIV disease, the severity of which has been associated with the presence of lipodystrophy $y^{4,33,223}$ and with greater weight gain following ART initiation ${ }^{12,65}$.

The avoidance of older ARTs that can induce lipoatrophy and central lipohypertrophy is also warranted to try and prevent fat alterations, whenever possible. In addition, close monitoring of weight gain and aggressive lifestyle interventions, such as healthy diet and regular exercise, could prevent the aggravation of lipohypertrophy by obesity and monitoring of waist circumference could allow earlier introduction of therapies to reduce VAT in PLWH. With improved understanding of how to reduce chronic inflammation, immune activation and gut barrier disruption in PLWH, new opportunities to prevent the contribution of these factors to lipodystrophy may emerge.

\section{Management}

Adopting a healthy lifestyle is the most effective and safest means to prevent and treat abdominal obesity in PLWH. This lifestyle should form part of the routine management strategy for all PLWH, particularly as overweight and obesity is increasingly common in the general population ${ }^{62,224}$. Judicious use of pharmacotherapy, including approved weight-reducing drugs and tesamorelin in appropriate individuals, can be effective, although patients should be carefully monitored as the long-term benefits and adverse effects remain uncertain.

\section{Lipoatrophy}

Facial lipoatrophy in patients treated with thymidine NRTIs is only partially reversed when patients undergo medication switching to remove the responsible drugs and may take months or longer to become clinically apparent ${ }^{225,226}$. Some patients with persistent lipoatrophy may benefit from plastic surgery. Initially, the patient's SAT was injected into the cheeks using the Coleman technique, but injection of poly-L-lactic acid has the highest efficacy in published studies and is FDA approved. In addition, poly-L-lactic acid is less frequently associated with local infection and poor patient satisfaction, is more durable and may improve quality of life (QOL). Calcium hydroxyapatite, which is also FDA approved, and hyaluronic acid, among other products, are also used but have less supporting evidence ${ }^{227}$; re-injection may be required within 1-2 years and can need further repetition ${ }^{41,228}$.

\section{Abdominal obesity}

The most common type of fat alteration in the current cART era in PLWH is central adiposity. Management of central adiposity in the general population is complicated and has an uncertain long-term efficacy. This management includes adopting a reduced-calorie diet and a sustained exercise programme, and can decrease total fat and VAT quantity in the short term as well as the risk of developing T2DM and cardiovascular disease $^{229}$. Similarly, the management of abdominal obesity in PLWH is also challenging. PLWH with abdominal obesity should undertake regular exercise and consume a healthy diet, although the long-term efficacy of these strategies in this population remains to be proven. Medication switching from a PI associated with abdominal obesity to either a non-NRTI or a PI with fewer associated fat mass complications is generally not effective in reducing $\mathrm{VAT}^{212}$. Whether INSTI-associated weight gain is related to increased clinical risks or is reversible is unknown. Accordingly, it is important to evaluate the reversibility of the effect of INSTIs and propose switching from these drugs as a therapeutic strategy.

Pharmacological therapy. Pharmacotherapy for the treatment of generalized obesity is effective in the general population but the long-term benefits of weightreducing drugs remains controversial as their ability to substantially reduce VAT is uncertain and adverse effects are common ${ }^{230}$.

The GLP1 agonist liraglutide is effective in reducing weight in individuals with obesity with or without $\mathrm{T}_{2} \mathrm{DM}^{231}$ and is associated with a reduction in the frequency of cardiovascular events in patients with T2DM ${ }^{232}$. However, this drug class has not been evaluated in PLWH. The insulin-sensitizing drug metformin causes a $10 \%$ reduction of VAT in PLWH with lipohypertrophy but is associated with worsening peripheral lipoatrophy in patients with mixed body shape phenotypes (that is, those with both lipoatrophy and lipohypertrophy) ${ }^{233}$. Whether metformin is effective in PLWH with glucose homeostasis abnormalities and abdominal obesity but without peripheral lipoatrophy has not been assessed. One study of recombinant leptin significantly decreased VAT in $\mathrm{PLWH}^{234}$. Testosterone replacement can decrease total fat mass in the general population but has an uncertain effect on reducing VAT and may increase cardiovascular disease risk in men $>65$ years of age ${ }^{235}$. In one study, testosterone replacement in hypogonadal male 
PLWH decreased DXA-derived trunk fat although there was no change in VAT assessed using CT, reflecting the need for accurate imaging modalities to assess therapeutic responses ${ }^{236}$. The treatments described above are not generally recommended for the management of abdominal obesity in PLWH.

\section{Modulation of the growth hormone axis}

HIV-negative (non-infected) persons with endocrine disorders associated with abdominal obesity, including growth hormone deficiency (GHD) and hypogonadism, can benefit from growth hormone $(\mathrm{GH})$ replacement. $\mathrm{GH}$ treatment has also been studied in people with abdominal obesity without $\mathrm{GHD}^{237-239}$. However, these studies are limited by short durations and infrequent use of accurate abdominal fat assessments. Indeed, although GH treatment reduced VAT in patients with GHD and abdominal obesity, follow-up has generally been too short to determine whether this effect is sustained and whether the risk of cardiovascular disease is reduced ${ }^{240}$.

Despite the limitations of these studies, owing to the short-term benefits of treatment in seronegative persons with GHD and the documented abnormalities of the GH/insulin-like growth factor 1 (IGF1) axis in treated $\mathrm{PLWH}^{241}$, GH replacement has also been studied in PLWH. GH/IGF1 disturbances in PLWH include a reduction in mean overnight $\mathrm{GH}$ levels resulting from decreased GH pulse amplitude, pulse width and trough GH levels, with no change in pulse frequency, and decreased $\mathrm{GH}$ response to $\mathrm{GH}$-releasing hormone-arginine stimulation. Furthermore, they are closely related to increased visceral adiposity ${ }^{242,243}$. In these studies, recombinant human GH significantly decreased VAT in the short-term compared with placebo ${ }^{24,245}$; however, glucose homeostasis was impaired and higher SAT reductions were observed in patients with baseline lipoatrophy than in those without lipoatrophy. These limitations were not affected by changing dosing schedules ${ }^{229,245}$. In addition, although VAT decreased with treatment, it returned to baseline levels following drug cessation. Based on the results of these trials, GH is not approved for the treatment of abdominal obesity in PLWH.

Other therapies targeting the $\mathrm{GH}$ pathway have been evaluated for the treatment of abdominal obesity in PLWH. GH-releasing factor (GHRF) has been shown to restore physiological control of $\mathrm{GH}$ secretion, maintain normal negative feedback of IGF1 on GH release, and limit GH-related toxicities in PLWH and lipohypertro$\mathrm{phy}^{246}$. In addition, synthetic GHRF was associated with increased baseline IGF1 levels within the normal range and with reductions in DXA-assessed trunk fat, albeit with a non-significant reduction in CT-assessed VAT of 9\%, in male PLWH with increased VAT (CT-assessed VAT of $\left.180 \mathrm{~cm}^{2}\right)^{247}$.

Tesamorelin, a synthetic human $\mathrm{GHRF}^{248}$, was demonstrated to decrease DXA-trunk fat by $9.2 \%$ and CT-assessed VAT by $15.7 \%$ compared with placebo in treated PLWH with abdominal obesity (diagnosed using anthropometric parameters associated with increased VAT in seronegative individuals ${ }^{219}$ ), impaired fasting glycaemia or diet-controlled T2DM. Importantly, SAT did not change between groups. No participants with baseline impaired fasting glycaemia developed T2DM. Interestingly, tesamorelin did not affect glucose homeostasis in HIV-seronegative persons without T2DM ${ }^{249}$.

In a pooled analysis of two studies of tesamorelin in PLWH with abdominal obesity ${ }^{250,251}$, VAT decreased by $15.4 \%$ in patients treated with tesamorelin, with an increase in IGF1 levels within the physiological range, reduced baseline triglycerides and a reduced total cholesterol to HDL cholesterol ratio in those who received tesamorelin compared with placebo ${ }^{251}$. In addition, a minimal increase in $\mathrm{HbA}_{1 \mathrm{c}}$ of unknown significance occurred in patients who received tesamorelin. Furthermore, QOL was reduced in persons on placebo and remained unchanged in treated participants ${ }^{251}$. During the 26-week extension phase of the trial, treated persons maintained the VAT reduction and improvements in trunk fat, waist circumference, triglycerides and IGF1. By contrast, VAT and trunk fat returned to baseline levels within 3 months in individuals who received tesamorelin during the first phase of the trial, but who switched to placebo during the extension phase. Adverse events were similar between groups ${ }^{251,252}$. Overall, $\sim 66 \%$ of tesamorelin-treated PLWH achieved a significant reduction of VAT. Further studies of tesamorelin have demonstrated that improvements in metabolic parameters (including triglyceride and adiponectin levels) and inflammatory markers (including a reduction in tissue plasminogen activator levels) were more likely to occur in tesamorelin responders than in non-responders ${ }^{253,254}$. Moreover, tesamorelin has also been shown to reduce liver fat and pretreatment elevated transaminases in responders ${ }^{255,256}$. Indeed, one study showed a significant reduction of hepatic fat and prevention of progression of fibrosis, without improvement in existing fibrosis, in treated PLWH with hepatic steatosis ${ }^{257}$. This finding is important as liver fibrosis is a predictor of mortality in persons with NAFLD, an emerging non-AIDS complication in $\mathrm{PLWH}^{258}$. Although participants in this trial were not selected for the presence of increased VAT, VAT was increased in all participants and decreased with treatment; $60-70 \%$ of all participants were receiving INSTI-based regimens, but it was not possible to differentiate responses between individuals who had previously received other cART regimens versus those only on currently recommended first-line drugs.

Tesamorelin is the only drug approved for the reduction of excess abdominal fat in PLWH with lipohypertrophy and is licensed in the USA, Canada and several other countries. Follow-up studies will clarify whether supraphysiological IGF1 levels are associated with increased cancer risk, as has been suggested for people without HIV ${ }^{259}$. However, the risk of cancer with tesamorelin may be low, as IGF1 generally increases within the normal range, and GH replacement in seronegative persons with GHD showed no association with malignancy if IGF1 levels remained within normal age-related ranges $^{260}$. Tesamorelin discontinuation is recommended in PLWH if IGF1 levels increase persistently by more 
than three standard deviations (Theratechnologies). In addition, tesamorelin cessation should be considered if de novo impaired glucose tolerance or T2DM develops. The long-term effects of tesamorelin on cardiometabolic outcomes, the optimal treatment duration and options for the long-term maintenance of decreased VAT are unknown.

\section{Surgery for weight reduction and buffalo hump}

Bariatric and metabolic surgery for individuals with severe obesity in the general population leads to improved longterm survival and QOL as well as to sustained weight loss and amelioration of obesity-related comorbidities ${ }^{261}$. Studies of bariatric surgery for severe generalized obesity $\left(\mathrm{BMI}>40 \mathrm{~kg} / \mathrm{m}^{2}\right)$ in PLWH are limited to small series and have reported uncertain effects on VAT. However, bariatric surgery might be effective for weight loss and to decrease metabolic complications in some patients ${ }^{262}$. After bariatric surgery, some cART-treated PLWH can have a detectable viral load and decreased exposure to some antiretroviral drugs and should be closely monitored after surgery ${ }^{263}$. The role of surgery specifically to remove excess VAT is unclear, with potential beneficial effects on weight but not metabolic health or adipokine secretion $^{264,265}$

Surgical lipectomy for functional or clinical indications of enlarged DCAT has been reported in small series with acceptable results and with at least 2 years of follow-up, although caution is urged because of increased recurrence rates ${ }^{266-268}$.

\section{Quality of life}

PLWH must be engaged in the cascade of HIV care to fully benefit from cART. In 2014, the Joint United Nations Program on HIV and AIDS set up the ' $90-90$ 90' targets to be achieved by 2020 (90-90-90: treatment for all) (that is, 90\% of PLWH diagnosed early after infection, 90\% linked and retained in care, and 90\% receiving and adhering to effective therapy). In 2018, $\sim 79 \%$ of PLWH knew their HIV status, $78 \%$ of those who knew their HIV status were accessing treatment and $86 \%$ of PLWH who were accessing treatment were virally suppressed, therefore keeping them alive and well and
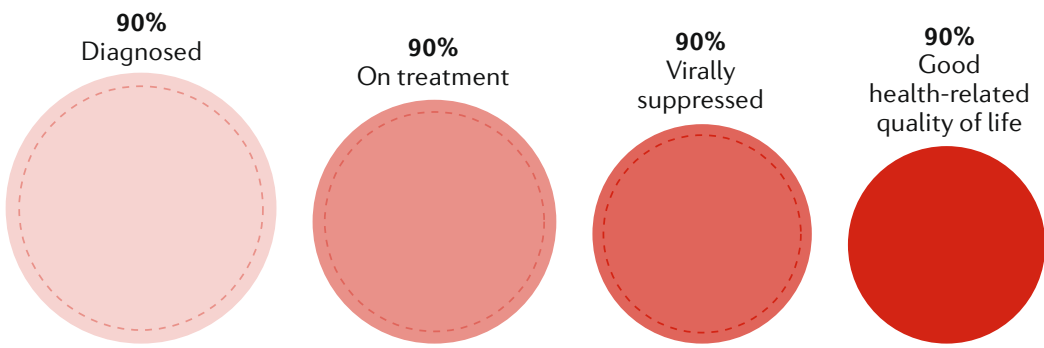

Fig. 5 | The 'fourth 90': proposed revision to the UNAIDS 90-90-90 targets. The Joint United Nations Program on HIV and AIDS set up the '90-90-90' targets to be achieved by 2020 ( $90 \%$ of people living with HIV diagnosed early, $90 \%$ linked and retained in care, and $90 \%$ receive and adhere to effective therapy). Lazarus et al. argue that there is an urgent need to contend with other challenges, including non-communicable diseases, mental health or HIV-related discrimination, and have proposed a fourth '90', that of achieving good health-related quality of life among $90 \%$ of those successfully treated for HIV ${ }^{270}$. Adapted from REF. ${ }^{270}$, CC BY 4.0 (https://creativecommons.org/licenses/ by/4.0/). preventing transmission of the virus ${ }^{269}$. Therefore, major progress has been made but there is still an important gap to reach the objectives.

Notably, the '90-90-90' targets are public health objectives and fail to address the health of PLWH as defined by the WHO as "a state of complete physical, mental and social well-being and not merely the absence of disease or infirmity". Indeed, some individuals argue that there is an urgent need to contend with other challenges, including non-communicable diseases, mental health and HIV-related discrimination, and have proposed a fourth '90' of achieving good health-related QOL (HRQOL) among 90\% of those successfully treated for $\mathrm{HIV}^{270}$ (FIG. 5).

Several QOL changes have been reported in PLWH. Indeed, PLWH with viral suppression have reported high levels of fatigue or energy loss, insomnia, sadness or depression, sexual dysfunction, and changes in body appearance, among other symptoms ${ }^{271}$. Lipoatrophy and lipohypertrophy have been associated with depression in adults as well as in older children and adolescents ${ }^{272,273}$. In addition, one cross-sectional study comparing $>3,000$ PLWH with $>7,000$ members of the general population in the UK reported that HRQOL scores were significantly lower in PLWH and remained low in those with viral suppression ${ }^{274}$.

After the advent of effective cART in the mid-tolate 1990s, lipodystrophy was generally considered as an unavoidable adverse effect of life-saving therapies. Of the fat alterations in PLWH, facial lipoatrophy is particularly stigmatizing and is suggestive of the use of anti-HIV drugs ${ }^{275,276}$. However, studies specifically assessing the effect of fat alterations on HRQOL are scarce, particularly in children (BOX 2), and the available QOL instruments do not allow a comparative assessment across countries, studies and populations. The most frequently used instruments used to assess QOL (such as the Short Form 36 (SF-36) ${ }^{277}$, the EuroQoL 5 Domains (EQ-5D) ${ }^{274}$ or the Medical Outcomes Study in Health Survey (MOS-HIV) ${ }^{278}$ ) do not specifically assess the effect of body composition changes; however, some QOL assessment tools specifically include questions regarding lipodystrophy, such as the lipodystrophyspecific Assessment of Body Change and Distress $(\mathrm{ABCD})^{279}$ and the HIV/AIDS-Targeted Quality of Life (HAT-QoL) ${ }^{280}$. Assessing HRQOL is challenging as it is subjective, multifaceted and includes psychological, physical, social and personal outcomes ${ }^{281}$. Interestingly, although most data from Brazil, Europe and the USA have suggested a negative effect of lipodystrophy on QOL ${ }^{282-285}$, this effect was not observed in one cross-sectional, hospital-based evaluation in Ethiopia ${ }^{286}$. By contrast, one study in Spain indicated that lipodystrophy is associated with social isolation, even years after the HIV diagnosis ${ }^{287}$. The negative effect of body fat changes seems to be even more critical in women whose self-perception of these changes was closely associated with a decrease in $\mathrm{QOL}^{288,289}$. However, one review of 23 studies demonstrated that the use of facial fillers and autologous fat transfer (Coleman intervention) were associated with improved QOL patient scores in $\mathrm{PLWH}^{281}$. 


\section{Outlook}

Despite advances in our understanding of the pathogenesis, consequences and treatment of fat alterations in PLWH, there is still much to be learned and new challenges have arisen that must be addressed in future research studies.

\section{Effect of HIV on adipose function}

Systemic inflammation and bacterial translocation in the gut lymphoid tissue, which may be influenced by changes in the gut microbiome, are likely important mechanisms for adipose dysfunction in PLWH. Improved understanding of the molecular mechanisms underlying these pathways could lead to targeted strategies to treat fat alterations in PLWH. In addition, the recognition of adipose tissue as a reservoir for HIV could have important consequences for the functional cure of HIV infection. Future studies that aim to alter the gut microbiota in PLWH, reduce bacterial translocation or affect systemic inflammation should also determine their effects on adipose function, including adipokine and cytokine secretion and free fatty acid uptake and release. In addition, strategies to eradicate HIV should also focus on HIV persistence in adipose tissue. Conversely, strategies aimed at decreasing fat mass and improving adipose function in PLWH should also assess the potential effects on the HIV reservoir in fat.

\section{Effect of cART on fat}

Although the metabolic adverse effects of antiretroviral therapies have improved markedly over the past 20 years, newer, well-tolerated medications, such as INSTIs and TAF, have been shown to lead to more weight gain than other agents. This finding poses challenges in the clinical care of PLWH given the efficacy, low toxicity and popularity of these medications. Accordingly, research evaluating the magnitude of these changes is important in addition to determining which patients are most likely to be affected (for example, the use of early biomarkers or demographic or clinical data) and the clinical consequences. Furthermore, strategies investigating the effect of switching from INSTIs or TAF on adipose volume and function in virally suppressed PLWH who have substantial fat alterations should be evaluated.

Understanding the changes in adipose tissue during the first 2 years after cART initiation and evaluation of the strategies to prevent this weight gain are also critical research topics. Prevention strategies could include patient counselling before cART initiation and other behavioural approaches, adjunctive medications or avoidance of ARTs most associated with weight gain in those at the highest risk. In addition, it has been suggested that HIV and some ARTs can induce fat fibrosis; thus, determining the reversibility of this fibrosis and, if partial, the possible remaining irreversible scar of adipose tissue, is essential.

\section{Ectopic fat in HIV and its health consequences}

As previously mentioned, ectopic expansion of fat deposits can also occur disproportionately in PLWH. Pancreatic and myocardial fat deposits in PLWH have not been well studied and require further research.

\section{Box 2 | Fat alterations in children with HIV}

Although children with HIV are commonly affected by body fat changes, studies assessing the effect of these changes using quality-of-life (QOL) tools are scarce. Disfigurement ${ }^{294}$, HIV status disclosure ${ }^{295}$ and stigmatization are commonly reported concerns in observational cohorts. Indeed, disclosure concerns have been suggested as a relevant target for interventions to decrease HIV-related stigma and increase healthrelated QOL in children. Body fat changes that affect the growth and well-being of children are well known and have a significant impact on the mental health of older children and adolescents, leading to poor self-image, depression and subsequent poor adherence to therapy ${ }^{273}$ However, QOL tools have not been specifically used to assess the effect of body fat changes in children. Every effort should be made to use antiretroviral therapies with no or limited fat toxicity. Owing to the delay of almost 5 years in the commercialization of a new drug and its license for use in children, children have been neglected with regard to drug innovation and have been exposed to offending drugs and, therefore, to drug-related adverse events ${ }^{296}$.

By contrast, liver fat (NAFLD or non-alcoholic steatohepatitis) continue to be a major focus of research, with several on-going trials (NCT03129113, NCT03913351, NCT03669133 and NCT02196831).

\section{Adipose alterations in an ageing HIV population}

In the general population, ageing is associated with increases in VAT, losses in SAT and accumulation of fat in ectopic sites. These fat changes are thought to be a major source of inflammation and immune activation and have a similar phenotype to those seen in PLWH. For older (generally $>50$ years of age) PLWH, these fat changes have been associated with physical frailty and cognitive decline ${ }^{290-292}$, suggesting that reversing fat alterations in older PLWH could also reduce the burden of these ageing-related comorbidities. Based on this hypothesis, trials investigating the effects of tesamorelin (NCT02572323), the GLP1 analogue liraglutide (NCT02743598) and exercise (NCT02663934) on cognitive function in PLWH are ongoing.

\section{Fat changes in low-/middle-income countries}

Most cases of HIV are in low-income and middleincome countries. In these countries, improvements in health care, sanitation and food availability have led to increases in lifespan among the general population, although urbanization and adoption of the Western (high-calorie) diet have led to increasing rates of obesity and obesity-related conditions ${ }^{293}$. In the context of these changes, PLWH in low-income and middle-income countries are surviving for longer where cART is available and are increasingly receiving new medications that are associated with weight gain (such as INSTIs). The next phase of research into this area will involve understanding both the global burden of obesity in PLWH and its consequences.

Published online: 18 June 2020 
1. Ghaben, A. L. \& Scherer, P. E. Adipogenesis and metabolic health. Nat. Rev. Mol. Cell Biol. 20 242-258 (2019)

2. Scheja, L. \& Heeren, J. The endocrine function of adipose tissues in health and cardiometabolic disease. Nat. Rev. Endocrinol. 15, 507-524 (2019).

3. Schosserer, M., Grillari, J., Wolfrum, C. \& Scheideler, M. Age-induced changes in white, brite, and brown adipose depots: a mini-review. Gerontology 64, 229-236 (2018).

4. Carr, A., Law, M. \& HIV Lipodystrophy Case Definition Study Group. An objective lipodystrophy severity grading scale derived from the lipodystrophy case definition score. J. Acquir. Immune Defic. Syndr. 33 , 571-576 (2003)

5. Saint-Marc, T. et al. A syndrome of peripheral fat wasting (lipodystrophy) in patients receiving long-term nucleoside analogue therapy. AIDS 13, 1659-1667 (1999).

6. NCD Risk Factor Collaboration (NCD-RisC). Trends in adult body-mass index in 200 countries from 1975 to 2014: a pooled analysis of 1698 population-based measurement studies with 19.2 million participants. Lancet 387, 1377-1396 (2016).

7. Martin, A. et al. Reversibility of lipoatrophy in HIV-infected patients 2 years after switching from a thymidine analogue to abacavir: the MITOX extension study. AIDS 18, 1029-1036 (2004)

8. Guaraldi, G. et al. The natural history of HIV-associated lipodystrophy in the changing scenario of HIV infection. HIV Med. 15, 587-594 (2014).

9. McComsey, G. A et al. Peripheral and central fat changes in subjects randomized to abacavir-lamivudine or tenofovir-emtricitabine with atazanavir-ritonavir or efavirenz: ACTC Study A5224s. Clin. Infect. Dis. 53, 185-196 (2011)

10. Hill, A., Waters, L. \& Pozniak, A. Are new antiretroviral treatments increasing the risks of clinical obesity? J. Virus Erad. 5, 41-43 (2019). A viewpoint on the clinical trials associating integrase inhibitor-based regimens and weight gain.

11. Venter, W. D. F. et al. Dolutegravir plus two different prodrugs of tenofovir to treat HIV. N. Engl. J. Med. 381, 803-815 (2019).

An important trial in ART-naive patients, showing more weight gain with dolutegravir-containing regimens, especially in combination with TAF than with the standard-care regimen.

12. Sax, P. E. et al. Weight gain following initiation of antiretroviral therapy: risk factors in randomized comparative clinical trials. Clin. Infect. Dis. https:/ doi.org/10.1093/cid/ciz999 (2019).

13. Smith, U. Abdominal obesity: a marker of ectopic fat accumulation. J. Clin. Invest. 125, 1790-1792 (2015).

14. Macallan, D. C. et al. Energy expenditure and wasting in human immunodeficiency virus infection. N. Engl. J. Med. 333, 83-88 (1995).

15. Macallan, D. C. Wasting in HIV infection and AIDS J. Nutr. 129, 238S-242S (1999)

16. Rivera, S., Briggs, W. Oian, D. \& Sattler, F. R. Levels of HIV RNA are quantitatively related to prior weight loss in HIV-associated wasting. J. Acquir. Immune Defic. Syndr. Hum. Retrovirol. 17, 411-418 (1998).

17. Melchior, J. C. et al. Resting energy expenditure is increased in stable, malnourished HIV-infected patients. Am. J. Clin. Nutr. 53, 437-441 (1991).

18. Grinspoon, S. et al. Body composition and endocrine function in women with acquired immunodeficiency syndrome wasting. J. Clin. Endocrinol. Metab. 82 , 1332-1337 (1997)

19. Kotler, D. \& Heymsfield, S. B. HIV infection: a model chronic illness for studying wasting diseases. Am. J. Clin. Nutr. 68, 519-520 (1998)

20. Kotler, D. P. Human immunodeficiency virus-related wasting: malabsorption syndromes. Semin. Oncol. 25 , 70-75 (1998).

21. Kotler, D. P. et al. Relative influences of sex, race, environment, and HIV infection on body composition in adults. Am. J. Clin. Nutr. 69, 432-439 (1999).

22. Visnegarwala, F. et al. Sex differences in the associations of HIV disease characteristics and body composition in antiretroviral-naive persons. Am. J. Clin. Nutr. 82, 850-856 (2005).

23. Caron-Debarle, M., Lagathu, C., Boccara, F. Vigouroux, C. \& Capeau, J. HIV-associated lipodystrophy: from fat injury to premature aging. Trends Mol. Med. 16, 218-229 (2010).

24. Herek, G., Saha, S. \& Burack, J. Stigma and psychological distress in people with HIV/AIDS Basic. Appl. Soc. Psychol. 35, 41-54 (2013).
25. Bacchetti, P. et al. Fat distribution in men with HIV infection. J. Acquir. Immune Defic. Syndr. $\mathbf{4 0}$ 121-131 (2005)

26. Study of Fat Redistribution and Metabolic Change in HIV Infection (FRAM). Fat distribution in women with HIV infection. J. Acquir. Immune Defic. Syndr. 42 562-571 (2006)

27. Beraldo, R. A. et al. Proposed ratios and cutoffs for the assessment of lipodystrophy in HIV-seropositive individuals. Eur. J. Clin. Nutr. 69, 274-278 (2015).

28. Domingo, P., Estrada, V., Lopez-Aldeguer, J., Villaroya, F. \& Martinez, E. Fat redistribution syndromes associated with HIV-1 infection and combination antiretroviral therapy. AIDS Rev. 14 112-123 (2012)

\section{A good review on the epidemiology and} mechanisms of HIV/ART-related lipodystrophy.

29. Paton, N. I., Earnest, A., Ng, Y. M., Karim, F. \& Aboulhab, J. Lipodystrophy in a cohort of human immunodeficiency virus-infected Asian patients: prevalence, associated factors, and psychological impact. Clin. Infect. Dis. 35, 1244-1249 (2002).

30. Alberti, K. G., George, M. M., Zimmet, P. \& Shaw, J. The metabolic syndrome - a new worldwide definition. Lancet 366, 1059-1062 (2005).

31. Finkelstein, J. L., Gala, P., Rochford, R., Glesby, M. J. \& Mehta, S. HIV/AIDS and lipodystrophy: implications for clinical management in resource-limited settings. J. Int. AIDS Soc. 18, 19033 (2015)

32. van Oosterhout, J. J. et al. Stavudine toxicity in adult longer-term ART patients in Blantyre, Malawi. PLoS One 7, e42029 (2012).

33. Han, S. H. et al. Prevalence of and risk factors for lipodystrophy among HIV-infected patients receiving combined antiretroviral treatment in the Asia-Pacific region: results from the TREAT Asia HIV Observational Database (TAHOD). Endocr. J. 58, 475-484 (2011).

34. Podzamczer, D. et al. How much fat loss is needed for lipoatrophy to become clinically evident? AIDS Res. Hum. Retroviruses 25, 563-567 (2009).

35. Coin, A. et al. Limb fat-free mass and fat mass reference values by dual-energy $\mathrm{X}$-ray absorptiometry (DEXA) in a 20-80 year-old Italian population. Clin. Nutr. 31, 506-511 (2012).

36. Mallon, P. W., Miller, J., Cooper, D. A. \& Carr, A Prospective evaluation of the effects of antiretroviral therapy on body composition in HIV-1 -infected men starting therapy. AIDS 17, 971-979 (2003).

37. Dube, M. P. et al. Long-term body fat outcomes in antiretroviral-naive participants randomized to nelfinavir or efavirenz or both plus dual nucleosides. Dual X-ray absorptiometry results from A5005s, a substudy of Adult Clinical Trials Group 384. J. Acquir. Immune Defic. Syndr. 45, 508-514 (2007) A study on the natural history of ART-related lipodystrophy.

38. Martin, A. \& Mallon, P. W. Therapeutic approaches to combating lipoatrophy: do they work? J. Antimicrob. Chemother. 55, 612-615 (2005)

39. Rockstroh, J. K. et al. Long-term treatment with raltegravir or efavirenz combined with tenofovir/ emtricitabine for treatment-naive human immunodeficiency virus-1-infected patients: 156-week results from STARTMRK. Clin. Infect. Dis. 53, 807-816 (2011).

40. World Health Organization. Rapid advice: antiretrovira therapy for HIV infection in adults and adolescents. WHO https://www.who.int/hiv/pub/arv/rapid advice art.pdf (41).

41. Lagathu, C. et al. Metabolic complications affecting adipose tissue, lipid and glucose metabolism associated with HIV antiretroviral treatment. Expert. Opin. Drug Saf. 18, 829-840 (2019).

42. Grunfeld, C. et al. Regional adipose tissue measured by MRI over 5 years in HIV-infected and control participants indicates persistence of HIV-associated lipoatrophy. AIDS 24, 1717-1726 (2010).

43. Schouten, J. et al. Cross-sectional comparison of the prevalence of age-associated comorbidities and their risk factors between HIV-infected and uninfected individuals: the AGEhIV cohort study. Clin. Infect. Dis. 59, 1787-1797 (2014)

44. Gelpi, M. et al. Prior exposure to thymidine analogues and didanosine is associated with long-lasting alterations in adipose tissue distribution and cardiovascular risk factors. AIDS 33, 675-683 (2018).

An important study highlighting the long-term role of thymidine NRTIs on fat distribution and metabolic disorders.

45. Gelpi, M. et al. Long-lasting alterations in fat distribution in PLWH exposed to thymidine analogues.
CROI https://www.croiconference.org/abstract/longlasting-alterations-fat-distribution-plwh-exposedthymidine-analogues/ (2019).

46. Bastard, J. P. et al. Diabetes and dyslipidaemia are associated with oxidative stress independently of inflammation in long-term antiretroviral-treated HIV-infected patients. Diabetes Metab. 45, 573-581 (2019).

47. Arrive, E. et al. Metabolic risk factors in young adults infected with HIV since childhood compared with the general population. PLoS One 13, e0206745 (2018).

48. de Waal, R., Cohen, K. \& Maartens, G. Systematic review of antiretroviral-associated lipodystrophy: lipoatrophy, but not central fat gain, is an antiretroviral adverse drug reaction. PLoS One 8, e63623 (2013).

49. Wohl, D. A. \& Brown, T. T. Management of morphologic changes associated with antiretroviral use in HIV-infected patients. J. Acquir. Immune Defic. Syndr. 49 (Suppl. 2), 93-100 (2008).

50. Mutimura, E., Stewart, A., Rheeder, P. \& Crowther, N. J. Metabolic function and the prevalence of lipodystrophy in a population of HIV-infected African subjects receiving highly active antiretroviral therapy. J. Acquir. Immune Defic. Syndr. 46, 451-455 (2007).

51. Moyle, G. et al. Epidemiology, assessment, and management of excess abdominal fat in persons with HIV infection. AIDS Rev. 12, 3-14 (2010).

52. Guaraldi, G. et al. Prevalence of and risk factors for pubic lipoma development in HIV-infected persons. J. Acquir. Immune Defic. Syndr. 45, 72-76 (2007).

53. Haubrich, R. H. et al. Metabolic outcomes in a randomized trial of nucleoside, nonnucleoside and protease inhibitor-sparing regimens for initial HIV treatment. AIDS 23, 1109-1118 (2009).

54. Vrouenraets, S. M. et al. Randomized comparison of metabolic and renal effects of saquinavir/ $r$ or atazanavir/r plus tenofovir/emtricitabine in treatmentnaive HIV-1-infected patients. HIV Med. 12, 620-631 (2011).

55. Moyle, G. J., Hardy, H., Farajallah, A., DeGrosky, M. \& McGrath, D. Comparison of body composition changes between atazanavir/ritonavir and lopinavir/ritonavir each in combination with tenofovir/emtricitabine in antiretroviral-naive patients with HIV-1 infection. Clin. Drug Investig. 34, 287-296 (2014).

56. McComsey, G. A. et al. Body composition changes after initiation of raltegravir or protease inhibitors: ACTG A5260s. Clin. Infect. Dis. 62, 853-862 (2016). A carefully performed ACTC trial analysing fat evolution in patients initiating ART with different regimens.

57. Bhagwat, P et al. Raltegravir is associated with greater abdominal fat increases after antiretroviral therapy initiation compared to protease inhibitors. Antivir. Ther. 21 (Suppl. 1), A9 (2016)

58. Grant, P. M. et al. Long-term body composition changes in antiretroviral-treated HIV-infected individuals. AIDS 30, 2805-2813 (2016).

59. Debroy, P. et al. Progressive increases in fat mass occur in adults living with HIV on antiretroviral therapy, but patterns differ by sex and anatomic depot. J. Antimicrob. Chemother. 74, 1028-1034 (2019).

60. Shah, R. V. et al. Visceral adiposity and the risk of metabolic syndrome across body mass index: the MESA Study. JACC Cardiovasc. Imaging 7 1221-1235 (2014).

61. Scherzer, R. et al. Decreased limb muscle and increased central adiposity are associated with 5-year all-cause mortality in HIV infection. AIDS 25, 1405-1414 (2011).

62. Koethe, J. R. et al. Rising obesity prevalence and weight gain among adults starting antiretroviral therapy in the United States and Canada. AIDS Res. Hum. Retroviruses 32, 50-58 (2016).

63. Coetzee, L. et al. HIV, antiretroviral therapy and non-communicable diseases in sub-Saharan Africa: empirical evidence from 44 countries over the period 2000 to 2016. J. Int. AIDS Soc. 22, e25364 (2019).

64. Lake, J. E. The fat of the matter: obesity and visceral adiposity in treated HIV infection. Curr. HIV/AIDS Rep. 14, 211-219 (2017).

65. Bakal, D. R. et al. Obesity following ART initiation is common and influenced by both traditional and HIV- 1 ART-specific risk factors. J. Antimicrob. Chemother. 73 2177-2185 (2018).

66. Menard, A. et al. Dolutegravir and weight gain an unexpected bothering side effect? AIDS 31 1499-1500 (2017).

67. Bourgi, K. et al. Greater weight gain among treatmentnaive persons starting integrase inhibitors. $\mathrm{CROI}$ 
https://www.croiconference.org/abstract/greaterweight-gain-among-treatment-naive-persons-startingintegrase-inhibitors/ (2019).

68. Bourgi, K. et al. Greater weight gain in treatment naive persons starting dolutegravir-based antiretroviral therapy. Clin. Infect. Dis. 70, 1267-1274 (2020)

69. Bourgi, K. et al. Weight gain among treatment-naïve persons with HIV starting integrase inhibitors compared to non-nucleoside reverse transcriptase inhibitors or protease inhibitors in a large observational cohort in the United States and Canada. J. Int. AIDS Soc. 23 e25484 (2020)

70. Rizzardo, S. et al. Dolutegravir monotherapy and body weight gain in antiretroviral naive patients. AIDS 33 1673-1674 (2019)

71. Couturier, J. et al. Adipocytes impair efficacy of antiretroviral therapy. Antivir. Res. 154, 140-148 (2018).

72. Gorwood, J. et al. The integrase inhibitors dolutegravi and raltegravir exert pro-adipogenic and profibrotic effects and induce insulin resistance in human/simian adipose tissue and human adipocytes. Clin. Infect. Dis. https://doi.org/10.1093/cid/ciaa2595804258 (2020).

73. Burns, J. E. et al. No overall change in the rate of weight gain after switching to an integrase-inhibitor in virologically suppressed adults with HIV. AIDS 34 , 109-114 (2020)

74. Lake, J. E. et al. Risk factors for weight gain following switch to integrase inhibitor-based antiretroviral therapy. Clin. Infect. Dis. https://doi.org/10.1093/ $\mathrm{cid} / \mathrm{ciaa} 177$ (2020).

75. Katlama, C. et al. Dual therapy combining raltegravir with etravirine maintains a high level of viral suppression over 96 weeks in long-term experienced HIV-infected individuals over 45 years on a PI-based regimen: results from the Phase II ANRS 163 ETRAL study. J. Antimicrob. Chemother. 74, 2742-2751 (2019).

76. Assoumou, L. et al. Impact of the reproductive/ hormonal status on weight, fat and insulin resistance in HIV-infected women switching from a PI regimen to dual raltegravir-etravirine therapy: results from the ANRS163-ETRAL trial at 48 and 96 weeks. HIV Med. 20 (Suppl. 9), 132 (2019).

77. Currier, J. S. \& Havlir, D. V. CROI 2019: complications and coinfections in HIV infection. Top. Antivir. Med. 27 34-40 (2019).

78. Guaraldi, G. et al. Epicardial adipose tissue is an independent marker of cardiovascular risk in HIV-infected patients. AIDS 25, 1199-1205 (2011)

79. Buggey, J. \& Longenecker, C. T. Heart fat in HIV marker or mediator of risk? Curr. Opin. HIV AIDS 12, 572-578 (2017).

80. Lo, J. et al. Increased epicardial adipose tissue volume in HIV-infected men and relationships to body composition and metabolic parameters. AIDS $\mathbf{2 4}$ 2127-2130 (2010)

81. Torriani, M., Hadigan, C., Jensen, M. E. \& Grinspoon, S. Psoas muscle attenuation measurement with computed tomography indicates intramuscular fat accumulation in patients with the HIV-lipodystrophy syndrome. J. Appl. Physiol. 95, 1005-1010 (2003)

82. Natsag J et al. HIV infection is associated with increased fatty infiltration of the thigh muscle with aging independent of fat distribution. PLoS One 12, e0169184 (2017).

83. Fourman, L. T. et al. Differential relationships of hepatic and epicardial fat to body composition in HIV. Physiol. Rep. 5, e13386 (2017).

84. Lemoine, M. et al. Diagnostic accuracy of noninvasive markers of steatosis, NASH, and liver fibrosis in HIV-monoinfected individuals at risk of nonalcoholic fatty liver disease (NAFLD): results from the ECHAM study. J. Acquir. Immune Defic. Syndr. 80, e86-e94 (2019)

85. Couturier, J. et al. Infectious SIV resides in adipose tissue and induces metabolic defects in chronically infected rhesus macaques. Retrovirology 13, 30 (2016)

86. Couturier, J. et al. Human adipose tissue as a reservoi for memory CD4 ${ }^{+} \mathrm{T}$ cells and HIV. AIDS 29, 667-674 (2015)

One of the first studies showing that adipose tissue is an HIV reservoir.

87. Damouche, A. et al. Adipose tissue is a neglected viral reservoir and an inflammatory site during chronic HIV and SIV infection. PLoS Pathog. 11, e 1005153 (2015)

One of the first studies showing that adipose tissue is a reservoir for human HIV and simian SIV.

88. Gupta, M. K. et al. HIV-1 Nef-induced cardiotoxicity through dysregulation of autophagy. Sci. Rep. 7, 8572 (2017).
89. Tugizov, S. M. et al. HIV-associated disruption of mucosal epithelium facilitates paracellular penetration by human papillomavirus. Virology 446, 378-388 (2013).

90. Agarwal, N. et al. HIV-1 Vpr induces adipose dysfunction in vivo through reciprocal effects on PPAR/GR co-regulation. Sci. Transl Med. 5, 213 ra164 (2013).

91. Delpierre, C. et al. Impact of HIV infection on total body composition in treatment-naive men evaluated by dual-energy X-ray absorptiometry comparison of 90 untreated HIV-infected men to 241 controls. J. Clin. Densitom. 10, 376-380 (2007).

92. Sharpstone, D. R., Ross, H. M. \& Gazzard, B. G The metabolic response to opportunistic infections in AIDS. AIDS 10, 1529-1533 (1996).

93. Giralt, $M$ et al. HIV-1 infection alters gene expression in adipose tissue, which contributes to HIV- 1/HAARTassociated lipodystrophy. Antivir. Ther. 11, 729-740 (2006).

94. Vidal, F. et al. Adipogenic/lipid, inflammatory, and mitochondrial parameters in subcutaneous adipose tissue of untreated HIV-1-infected long-term nonprogressors: significant alterations despite low viral burden. J. Acquir. Immune Defic. Syndr. 61, 131-137 (2012).

95. Das, $\mathrm{S}$ et al. In treatment-naive and antiretroviraltreated subjects with HIV, reduced plasma adiponectin is associated with a reduced fractional clearance rate of VLDL, IDL and LDL apolipoprotein B-100. Diabetologia 49, 538-542 (2006).

96. Garrabou, G. et al. Mitochondrial damage in adipose tissue of untreated HIV-infected patients. AIDS 25 , 165-170 (2011).

97. De Pauw, A., Tejerina, S., Raes, M., Keijer, J. \& Arnould, T. Mitochondrial (dys)function in adipocyte (de)differentiation and systemic metabolic alterations. Am. J. Pathol. 175, 927-939 (2009).

98. Gorwood, J. et al. Impact of HIV/simian immunodeficiency virus infection and viral proteins on adipose tissue fibrosis and adipogenesis. AIDS 33, 953-964 (2019).

A study indicating the deleterious impact of HIV and SIV on fat fibrosis.

99. Utay, N. S. et al. Telmisartan therapy does not improve lymph node or adipose tissue fibrosis more than continued antiretroviral therapy alone. J. Infect. Dis. 217, 1770-1781 (2018)

100. Koethe, J. R. Adipose tissue in HIV infection. Compr Physiol. 7, 1339-1357 (2017) An excellent general review on the role of HIV in adipose tissue.

101. Pallikkuth, S. \& Mohan, M. Adipose tissue: sanctuary for HIV/SIV persistence and replication. Trends Microbiol. 23, 748-750 (2015).

102. Balasubramanyam, A. et al. Effects of transgenic expression of HIV-1 Vpr on lipid and energy metabolism in mice. Am. J. Physiol. Endocrinol. Metab. 292 E40-E48 (2007).

103. Shrivastav, S. et al. Human immunodeficiency virus (HIV)-1 viral protein R suppresses transcriptional activity of peroxisome proliferator-activated receptor- $\gamma$ and inhibits adipocyte differentiation: implications for HIV-associated lipodystrophy. Mol. Endocrinol. 22, 234-247 (2008)

104. Otake, K. et al. HIV-1 Nef protein in the nucleus influences adipogenesis as well as viral transcription through the peroxisome proliferator-activated receptors. AIDS 18, 189-198 (2004).

105. Cotter, E. J., Chew, N., Powderly, W. G. \& Doran, P. P. HIV type 1 alters mesenchymal stem cell differentiation potential and cell phenotype ex vivo. AIDS Res. Hum. Retroviruses 27 , 187-199 (2011).

106. Diaz-Delfin, J., Domingo, P., Wabitsch, M., Giralt, M. \& Villarroya, F. HIV-1 Tat protein impairs adipogenesis and induces the expression and secretion of proinflammatory cytokines in human SGBS adipocytes. Antivir. Ther. 17, 529-540 (2012).

107. Perez-Matute, P., Perez-Martinez, L., Blanco, J. R. $\&$ Oteo, J. A. Role of mitochondria in HIV infection and associated metabolic disorders: focus on nonalcoholic fatty liver disease and lipodystrophy syndrome. Oxid. Med Cell Longev. 2013, 493413 (2013).

108. Fabbrini, E. et al. Association between specific adipose tissue $\mathrm{CD}^{+}{ }^{+}$-cell populations and insulin resistance in obese individuals. Gastroenterology 145, 366-374. e 1-3 (2013).

109. Feuerer, M. et al. Lean, but not obese, fat is enriched for a unique population of regulatory T cells that affect metabolic parameters. Nat. Med. 15, 930-939 (2009).
110. Nishimura, S. et al. CD8+ effector T cells contribute to macrophage recruitment and adipose tissue inflammation in obesity. Nat. Med. 15, 914-920 (2009).

111. Pandolfi, J. B. et al. ATP-induced inflammation drives tissue-resident Th17 cells in metabolically unhealthy obesity. J. Immunol. 196, 3287-3296 (2016).

112. Shikuma, C. M. et al. The role of HIV and monocytes/ macrophages in adipose tissue biology. J. Acquir. Immune Defic. Syndr. 65, 151-159 (2014).

113. Hotamisligil, G. S. et al. IRS-1-mediated inhibition of insulin receptor tyrosine kinase activity in TNF-alphaand obesity-induced insulin resistance. Science 271, 665-668 (1996)

114. Lumeng, C. N., Bodzin, J. L. \& Saltiel, A. R. Obesity induces a phenotypic switch in adipose tissue macrophage polarization. J. Clin. Invest 117 175-184 (2007).

115. Weisberg, S. P. et al. Obesity is associated with macrophage accumulation in adipose tissue. J. Clin. Invest. 112, 1796-1808 (2003).

116. Gao, D. et al. Interleukin-1 $\beta$ mediates macrophageinduced impairment of insulin signaling in human primary adipocytes. Am. J. Physiol. Endocrinol. Metab. 307, E289-E304 (2014).

117. Lumeng, C. N., Deyoung, S. M. \& Saltiel, A. R. Macrophages block insulin action in adipocytes by altering expression of signaling and glucose transport proteins. Am. J. Physiol. Endocrinol. Metab. 292, E166-E174 (2007).

118. Jan, V. et al. Altered fat differentiation and adipocytokine expression are inter-related and linked to morphological changes and insulin resistance in HIV-1-infected lipodystrophic patients. Antivir. Ther. 9 555-564 (2004).

119. Damouche, A. et al. High proportion of PD-1-expressing $\mathrm{CD}^{+} \mathrm{T}$ cells in adipose tissue constitutes an immunomodulatory microenvironment that may support HIV persistence. Eur. J. Immunol. 47, 2113-2123 (2017).

120. Koethe, J. R. et al. Adipose tissue is enriched for activated and late-differentiated $C D 8^{+} T$ cells and shows distinct $\mathrm{CD} 8^{+}$receptor usage, compared with blood in HIV-infected persons. J. Acquir. Immune Defic. Syndr. 77, e14-e21 (2018)

121. Brown, T. T. et al. Antiretroviral therapy and the prevalence and incidence of diabetes mellitus in the multicenter AIDS cohort study. Arch. Intern. Med. 165, 1179-1184 (2005).

122. Capeau, J. et al. Ten-year diabetes incidence in 1046 HIV-infected patients started on a combination antiretroviral treatment. AIDS 26, 303-314 (2012).

123. De Wit, $\mathrm{S}$, et al. Incidence and risk factors for new-onset diabetes in HIV-infected patients: the Data Collection on Adverse Events of Anti-HIV Drugs (D:A:D) study. Diabetes Care 31, 1224-1229 (2008).

124. Guaraldi, G. et al. CD8 T-cell activation is associated with lipodystrophy and visceral fat accumulation in antiretroviral therapy-treated virologically suppressed HIV-infected patients. J. Acquir. Immune Defic. Syndr. 64, 360-366 (2013)

125. McDonnell, W. J. et al. High CD8 T-cell receptor clonality and altered CDR3 properties are associated with elevated isolevuglandins in adipose tissue during diet-induced obesity. Diabetes 67, 2361-2376 (2018).

126. Focosi, D., Bestagno, M., Burrone, O. \& Petrini, M $\mathrm{CD} 57^{+} \mathrm{T}$ lymphocytes and functional immune deficiency. J. Leukoc. Biol. 87, 107-116 (2010).

127. Palmer, B. E., Blyveis, N., Fontenot, A. P. \& Wilson, C. C. Functional and phenotypic characterization of $\mathrm{CD} 57^{+} \mathrm{CD} 4^{+} \mathrm{T}$ cells and their association with HIV-1-induced T cell dysfunction. J. Immunol. 175 , 8415-8423 (2005).

128. Wanjalla, C. N. et al. Adipose tissue in persons with $\mathrm{HIV}$ is enriched for $\mathrm{CD} 4^{+} \mathrm{T}$ effector memory and $\mathrm{t}$ effector memory RA+ cells, which show higher CD69 expression and CD57, CX3CR1, GPR56 co-expression with increasing glucose intolerance. Front. Immunol. 10, 408 (2019).

129. Gordon, C. L. et al. Induction and maintenance of CX3CR 1 -intermediate peripheral memory $\mathrm{CD} 8^{+} \mathrm{T}$ cells by persistent viruses and vaccines. Cell Rep. 23 768-782 (2018)

130. Nishimura, M. et al. Dual functions of fractalkine/CX3C ligand 1 in trafficking of perforin ${ }^{+} /$granzyme $\mathrm{B}^{+}$cytotoxic effector lymphocytes that are defined by CX3CR 1 expression. J. Immunol. 168, 6173-6180 (2002)

131. Pachnio, A. et al. Cytomegalovirus infection leads to development of high frequencies of cytotoxic virusspecific $\mathrm{CD}^{+} \mathrm{T}$ cells targeted to vascular endothelium PLoS Pathog. 12, e1005832 (2016). 
132. Peng, Y. M. et al. Specific expression of GPR56 by human cytotoxic lymphocytes. J. Leukoc. Biol. 90 735-740 (2011)

133. Bastard, J. P. et al. Association between altered expression of adipogenic factor SREBP1 in lipoatrophic adipose tissue from HIV-1-infected patients and abnormal adipocyte differentiation and insulin resistance. Lancet 359, 1026-1031 (2002).

134. Johnson, J. A. et al. Increased systemic and adipose tissue cytokines in patients with HIV-associated lipodystrophy. Am. J. Physiol. Endocrinol. Metab. 286 E261-E271 (2004)

135. Kannisto, K. et al. Expression of adipogenic transcription factors, peroxisome proliferator-activated receptor gamma co-activator 1, IL-6 and CD45 in subcutaneous adipose tissue in lipodystrophy associated with highly active antiretroviral therapy. AIDS 17, 1753-1762 (2003).

136. Lihn, A. S. et al. Increased expression of TNF- $\alpha$, IL-6, and IL-8 in HALS: implications for reduced adiponectin expression and plasma levels. Am. J. Physiol. Endocrinol. Metab. 285, E1072-E1080 (2003).

137. Podzamczer, D. et al. Less lipoatrophy and better lipid profile with abacavir as compared to stavudine: 96-week results of a randomized study. J. Acquir. Immune Defic. Syndr. 44, 139-147 (2007).

138. Nolan, D. et al. Mitochondrial DNA depletion and morphologic changes in adipocytes associated with nucleoside reverse transcriptase inhibitor therapy. AIDS 17, 1329-1338 (2003).

139. Hammond, E., Nolan, D., James, I., Metcalf, C. \& Mallal, S. Reduction of mitochondrial DNA content and respiratory chain activity occurs in adipocytes within 6-12 months of commencing nucleoside reverse transcriptase inhibitor therapy. AIDS 18, 815-817 (2004).

140. De Luca, A. et al. Mitochondrial DNA haplogroups and incidence of lipodystrophy in HIV-infected patients on long-term antiretroviral therapy. J. Acquir. Immune Defic. Syndr. 59, 113-120 (2012).

141. Hulgan, T. et al. European mitochondrial DNA haplogroups and metabolic changes during antiretroviral therapy in AIDS clinical trials group study A5142. AIDS 25, 37-47 (2011)

142. Nolan, D., Hammond, E., James, I., McKinnon, E. \& Mallal, S. Contribution of nucleoside-analogue reverse transcriptase inhibitor therapy to lipoatrophy from the population to the cellular level. Antivir. Ther 8, 617-626 (2003)

143. Mallal, S. A., John, M., Moore, C. B., James, I. R. \& McKinnon, E. J. Contribution of nucleoside analogue reverse transcriptase inhibitors to subcutaneous fat wasting in patients with HIV infection. AIDS 14, 1309-1316 (2000)

144. Chen, C. H. \& Cheng, Y. C. Delayed cytotoxicity and selective loss of mitochondrial DNA in cells treated with the anti-human immunodeficiency virus compound 2',3'-dideoxycytidine. J. Biol. Chem. 264 11934-11937 (1989)

145. Chen, C. H., Vazquez-Padua, M. \& Cheng, Y. C. Effect of anti-human immunodeficiency virus nucleoside analogs on mitochondrial DNA and its implication for delayed toxicity. Mol. Pharmacol. 39 625-628 (1991)

146. Cherry, C. L. et al. Exposure to dideoxynucleosides is reflected in lowered mitochondrial DNA in subcutaneous fat. J. Acquir. Immune Defic. Syndr. 30, 271-277 (2002)

147. Walker, U. A. et al. Evidence of nucleoside analogue reverse transcriptase inhibitor-associated genetic and structural defects of mitochondria in adipose tissue of HIV-infected patients. J. Acquir. Immune Defic. Syndr. 29, 117-121 (2002).

148. Pace, C. S. et al. Mitochondrial proliferation, DNA depletion and adipocyte differentiation in subcutaneous adipose tissue of HIV-positive HAART recipients. Antivir. Ther. 8, 323-331 (2003).

149. Walker, U. A., Setzer, B. \& Venhoff, N. Increased long-term mitochondrial toxicity in combinations of nucleoside analogue reverse-transcriptase inhibitors. AIDS 16, 2165-2173 (2002).

150. Hendrickson, S. L. et al. Mitochondrial DNA haplogroups influence lipoatrophy after highly active antiretroviral therapy. J. Acquir. Immune Defic. Syndr. 51, 111-116 (2009).

151. Gallego-Escuredo, J. M. et al. Differentially altered molecular signature of visceral adipose tissue in HIV-1-associated lipodystrophy. J. Acquir. Immune Defic. Syndr. 64, 142-148 (2013).

152. Hadigan, C. et al. Depot-specific regulation of glucose uptake and insulin sensitivity in HIV-lipodystrophy.
Am. J. Physiol. Endocrinol. Metab. 290, E289-E298 (2006).

153. Leroyer, S. et al. Glyceroneogenesis is inhibited through HIV protease inhibitor-induced inflammation in human subcutaneous but not visceral adipose tissue. J. Lipid Res. 52, 207-220 (2011).

154. Torriani, M. et al. Dysfunctional subcutaneous fat with reduced Dicer and Brown adipose tissue gene expression in HIV-infected patients. J. Clin. Endocrinol. Metab. 101, 1225-1234 (2016)

155. Mori, M. A. et al. Altered miRNA processing disrupts brown/white adipocyte determination and associates with lipodystrophy. J. Clin. Invest. 124, 3339-3351 (2014).

156. Srinivasa, S. et al. Brief Report: adipogenic expression of brown fat genes in HIV and HIV-related parameters. J. Acquir. Immune Defic. Syndr. 82, 491-495 (2019).

157. Walker, U. A. et al. Zidovudine induces visceral mitochondrial toxicity and intra-abdominal fat gain in a rodent model of lipodystrophy. Antivir. Ther. 19 783-792 (2014).

158. Mallon, P. W. et al. Buffalo hump seen in HIV-associated lipodystrophy is associated with hyperinsulinemia but not dyslipidemia. J. Acquir. Immune Defic. Syndr. 38 156-162 (2005)

159. Mori, M. A. et al. Role of microRNA processing in adipose tissue in stress defense and longevity. Cell Metab. 16, 336-347 (2012).

160. Thomou, T. et al. Adipose-derived circulating miRNAs regulate gene expression in other tissues. Nature 542, 450-455 (2017)

161. Bereziat, V. et al. LMNA mutations induce a noninflammatory fibrosis and a brown fat-like dystrophy of enlarged cervical adipose tissue. Am. J. Pathol. 179 2443-2453 (2011)

162. Guallar, J. P. et al. Differential gene expression indicates that 'buffalo hump' is a distinct adipose tissue disturbance in HIV-1-associated lipodystrophy. AIDS 22, 575-584 (2008).

163. Giralt, M., Domingo, P. \& Villarroya, F. Adipose tissue biology and HIV-infection. Best Pract. Res. Clin. Endocrinol. Metab. 25, 487-499 (2011). A good review on the effect of ART on adipocytes and adipose tissue, resulting in lipodystrophy.

164. Caron, M. et al. Some HIV protease inhibitors alter lamin $\mathrm{A} / \mathrm{C}$ maturation and stability, SREBP- 1 nuclear localization and adipocyte differentiation. AIDS 17 2437-2444 (2003).

165. Afonso, P. et al. LMNA mutations resulting in lipodystrophy and HIV protease inhibitors trigger vascular smooth muscle cell senescence and calcification: role of ZMPSTE24 downregulation. Atherosclerosis 245, 200-211 (2016).

166. Caron, M. et al. Human lipodystrophies linked to mutations in A-type lamins and to HIV protease inhibitor therapy are both associated with prelamin A accumulation, oxidative stress and premature cellular senescence. Cell Death Differ. 14, 1759-1767 (2007).

167. Capel, E., Auclair, M., Caron-Debarle, M. \& Capeau, J. Effects of ritonavir-boosted darunavir, atazanavir and lopinavir on adipose functions and insulin sensitivity in murine and human adipocytes. Antivir. Ther. 17. 549-556 (2012)

168. Jones, S. P., Waitt, C., Sutton, R., Back, D. J. \& Pirmohamed, M. Effect of atazanavir and ritonavir on the differentiation and adipokine secretion of human subcutaneous and omental preadipocytes. AIDS 22 1293-1298 (2008)

169. El Hadri, K. et al. In vitro suppression of the lipogenic pathway by the nonnucleoside reverse transcriptase inhibitor efavirenz in 3T3 and human preadipocytes or adipocytes. J. Biol. Chem. 279, 15130-15141 (2004).

170. Minami, R. et al. Comparison of the influence of four classes of HIV antiretrovirals on adipogeni differentiation: the minimal effect of raltegravir and atazanavir. J. Infect. Chemother. 17, 183-188 (2011).

171. Moure, R. et al. Impact of elvitegravir on human adipocytes: alterations in differentiation, gene expression and release of adipokines and cytokines. Antivir. Res. 132, 59-65 (2016)

172. Perez-Matute, P., Perez-Martinez, L., Blanco, J. R. ¿ Oteo, J. A. Neutral actions of raltegravir on adipogenesis, glucose metabolism and lipolysis in 3T3-L1 adipocytes. Curr. HIV Res. 9, 174-179 (2011).

173. Bastard, J. P. et al. Subcutaneous adipose tissue modifications induced by a switch to dual raltegravirmaraviroc therapy in controlled HIV-infected patients: a sub-study of the ANRS-ROCnRAL157 clinical trial. HIV Med. 20 (Suppl. 9), 128-129 (2019).
174. Dupin, N. et al. HIV and antiretroviral drug distribution in plasma and fat tissue of HIV-infected patients with lipodystrophy. AIDS 16, 2419-2424 (2002).

175. Madelain, V. et al. Impact of obesity on antiretroviral pharmacokinetics and immuno-virological response in HIV-infected patients: a case-control study. J. Antimicrob. Chemother. 72, 1137-1146 (2017).

176. Zhang, H. et al. Human gut microbiota in obesity and after gastric bypass. Proc. Natl Acad. Sci. USA 106, 2365-2370 (2009).

177. Karlsson, F. H. et al. Symptomatic atherosclerosis is associated with an altered gut metagenome. Nat. Commun. 3, 1245 (2012)

178. Jie, Z. et al. The gut microbiome in atherosclerotic cardiovascular disease. Nat. Commun. 8, 845 (2017)

179. Hansen, T. H., Gobel, R. J., Hansen, T. \& Pedersen, O. The gut microbiome in cardio-metabolic health Genome Med. 7, 33 (2015).

180. Qin, J. et al. A metagenome-wide association study of gut microbiota in type 2 diabetes. Nature $\mathbf{4 9 0}$, 55-60 (2012).

181. Dillon, S. M., Frank, D. N. \& Wilson, C. C. The gut microbiome and HIV-1 pathogenesis: a two-way street. AIDS 30, 2737-2751 (2016).

182. Vesterbacka, J. et al. Richer gut microbiota with distinct metabolic profile in HIV infected elite controllers. Sci. Rep. 7, 6269 (2017).

183. Sandler, N. G. \& Douek, D. C. Microbial translocation in HIV infection: causes, consequences and treatment opportunities. Nat. Rev. Microbiol. 10, 655-666 (2012).

184. Kewenig, S. et al. Rapid mucosal CD4 ${ }^{+}$T-cell depletion and enteropathy in simian immunodeficiency virusinfected rhesus macaques. Gastroenterology 116 1115-1123 (1999).

185. Brenchley, J. M. et al. CD4 ${ }^{+} \mathrm{T}$ cell depletion during all stages of HIV disease occurs predominantly in the gastrointestinal tract. J. Exp. Med. 200, 749-759 (2004).

186. Ellis, C. L. et al. Molecular characterization of stool microbiota in HIV-infected subjects by panbacterial and order-level $16 \mathrm{~S}$ ribosomal DNA (rDNA) quantification and correlations with immune activation. J. Acquir. Immune Defic. Syndr. 57, 363-370 (2011)

187. Gori, A. et al. Early impairment of gut function and gut flora supporting a role for alteration of gastrointestinal mucosa in human immunodeficiency virus pathogenesis. J. Clin. Microbiol. 46, 757-758 (2008).

188. Mehandru, S. et al. Primary HIV-1 infection is associated with preferential depletion of $\mathrm{CD}^{+} \mathrm{T}$ lymphocytes from effector sites in the gastrointestinal tract. J. Exp. Med. 200, 761-770 (2004).

189. Merlini, E. et al. Evidence for polymicrobic flora translocating in peripheral blood of HIV-infected patients with poor immune response to antiretroviral therapy. PLoS One 6, e18580 (2011).

190. Dillon, S. M. et al. An altered intestinal mucosal microbiome in HIV-1 infection is associated with mucosal and systemic immune activation and endotoxemia. Mucosal Immunol. 7, 983-994 (2014).

191. Gootenberg, D. B., Paer, J. M., Luevano, J. M. \& Kwon, D. S. HIV-associated changes in the enteric microbial community: potential role in loss of homeostasis and development of systemic inflammation. Curr. Opin. Infect. Dis. 30, 31-43 (2017).

192. Lozupone, C. A. et al. HIV-induced alteration in gut microbiota: driving factors, consequences, and effects of antiretroviral therapy. Gut Microbes 5, 562-570 (2014).

193. Monaco, C. L. et al. Altered virome and bacterial microbiome in human immunodeficiency virusassociated acquired immunodeficiency syndrome. Cell Host Microbe 19, 311-322 (2016).

194. Mutlu, E. A. et al. A compositional look at the human gastrointestinal microbiome and immune activation parameters in HIV infected subjects. PLoS Pathog. 10 e1003829 (2014).

195. Noguera-Julian, M. et al. Gut microbiota linked to sexual preference and HIV infection. EBioMedicine 5, 135-146 (2016)

196. Nowak, P. et al. Gut microbiota diversity predicts immune status in HIV-1 infection. AIDS 29 2409-2418 (2015).

197. Sze, M. A. \& Schloss, P. D. Looking for a signal in the noise: revisiting obesity and the microbiome. MBio 7 e01018-16 (2016)

198. Vazquez-Castellanos, J. F. et al. Altered metabolism of gut microbiota contributes to chronic immune activation in HIV-infected individuals. Mucosal Immunol. 8, 760-772 (2015). 
199. Vujkovic-Cvijin, I. et al. Dysbiosis of the gut microbiota is associated with HIV disease progression and tryptophan catabolism. Sci. Transl Med. 5, 193ra191 (2013).

200. Yu, G., Fadrosh, D., Ma, B., Ravel, J. \& Goedert, J. J. Anal microbiota profiles in HIV-positive and HIV-negative MSM. AIDS 28, 753-760 (2014).

201. Handley, S. A. et al. Pathogenic simian immunodeficiency virus infection is associated with expansion of the enteric virome. Cell 151, 253-266 (2012).

202. Dubourg, G., Surenaud, M., Levy, Y., Hue, S. \& Raoult, D. Microbiome of HIV-infected people. Microb. Pathog. 106, 85-93 (2017).

203. Cani, P. D. et al. Metabolic endotoxemia initiates obesity and insulin resistance. Diabetes 56, 1761-1772 (2007)

204. El Kamari, V. et al. Lower pretreatment gut integrity is independently associated with fat gain on antiretroviral therapy. Clin. Infect. Dis. 68 1394-1401 (2019).

205. Al-Sadi, R., Guo, S., Ye, D., Rawat, M. \& Ma, T. Y. TNF- $\alpha$ modulation of intestinal tight junction permeability is mediated by NIK/IKK-alpha axis activation of the canonical NF-kB pathway. Am. J. Pathol. 186, 1151-1165 (2016).

206. Chang, C. C. et al. Lipopolysaccharide promoted proliferation and adipogenesis of preadipocytes through JAK/STAT and AMPK-regulated CPLA2 expression. Int. J. Med. Sci. 16, 167-179 (2019).

207. Ortiz, A. M. \& Brenchley, J. M. Microbial translocation translating simian immunodeficiency virus to HIV. Curr. Opin. HIV AIDS 13, 15-21 (2018).

208. Brown, R. J. et al. The diagnosis and management of lipodystrophy syndromes: a multi-society practice guideline. J. Clin. Endocrinol. Metab. 101, 4500-4511 (2016).

An international practice guideline for the management of lipodystrophies.

209. Tien, P. C. et al. The study of fat redistribution and metabolic change in HIV infection (FRAM): methods, design, and sample characteristics. Am. J. Epidemiol. 163, 860-869 (2006)

210. Brown, T. T. et al. Fat distribution and longitudinal anthropometric changes in HIV-infected men with and without clinical evidence of lipodystrophy and HIV-uninfected controls: a substudy of the Multicenter AIDS Cohort Study. AIDS Res. Ther. 6, 8 (2009). A longitudinal evaluation of fat distribution in Multicenter AIDS Cohort Study patients with or without HIV

211. Joy, T. et al. Relation of body composition to body mass index in HIV-infected patients with metabolic abnormalities. J. Acquir. Immune Defic. Syndr. 47, 174-184 (2008)

212. Lake, J. E. et al. Practical review of recognition and management of obesity and lipohypertrophy in human immunodeficiency virus infection. Clin. Infect. Dis. 64 1422-1429 (2017)

\section{A study on GHD in PLWH}

213. Gulizia, R. et al. Proven intra and interobserver reliability in the echographic assessments of body fat changes related to HIV associated Adipose Redistribution Syndrome (HARS). Curr. HIV Res. 6 , 276-278 (2008)

214. Gulizia, R. et al. Comparability of echographic and tomographic assessments of body fat changes related to the HIV associated adipose redistribution syndrome (HARS) in antiretroviral treated patients. Ultrasound Med. Biol. 34, 1043-1048 (2008)

215. Freitas, P. et al. Assessment of body fat composition disturbances by bioimpedance analysis in HIV-infected adults. J. Endocrinol. Invest. 34, e321-e329 (2011).

216. Law, M., Puls, R., Cheng, A. K., Cooper, D. A. \& Carr, A. Evaluation of the HIV lipodystrophy case definition in a placebo-controlled, 144-week study in antiretroviral-naive adults. Antivir. Ther. 11, 179-186 (2006).

217. Guidelines (2013) for Managing Overweight and Obesity in Adults. reface to the Expert Panel Report (comprehensive version which includes systematic evidence review, evidence statements, and recommendations). Obesity 22 (Suppl. 2), S40 (2014).

218. Falutz, J., Rosenthall, L., Kotler, D., Zona, S. \& Guaraldi, G. Surrogate markers of visceral adipose tissue in treated HIV-infected patients: accuracy of waist circumference determination. HIV Med. 15 98-107 (2014).

219. Lemieux, S., Prud'homme, D., Bouchard, C., Tremblay, A. \& Despres, J. P. A single threshold value of waist girth identifies normal-weight and overweight subjects with excess visceral adipose tissue. Am. $J$. Clin. Nutr. 64, 685-693 (1996).

220. Despres, J. P \& Lamarche, B. Effects of diet and physical activity on adiposity and body fat distribution implications for the prevention of cardiovascular disease. Nutr. Res. Rev. 6, 137-159 (1993).

221. Leite, T. F. et al. Reduction of HIV-1 reservoir size and diversity after 1 year of CART among Brazilian individuals starting treatment during early stages of acute infection. Front. Microbiol. 10, 145 (2019).

222. Whitney, J. B. et al. Prevention of SIVmac251 reservoi seeding in rhesus monkeys by early antiretroviral therapy. Nat. Commun. 9, 5429 (2018).

223. Tsiodras, S., Perelas, A., Wanke, C. \& Mantzoros, C. S. The HIV-1/HAART associated metabolic syndrome novel adipokines, molecular associations and therapeutic implications. J. Infect. 61, 101-113 (2010).

224. Malaza, A., Mossong, J., Barnighausen, T. \& Newell, M. L. Hypertension and obesity in adults living in a high HIV prevalence rural area in South Africa. PLoS One 7, e47761 (2012).

225. McComsey, G. A. et al. Improvement in lipoatrophy associated with highly active antiretroviral therapy in human immunodeficiency virus-infected patients switched from stavudine to abacavir or zidovudine: the results of the TARHEEL study. Clin. Infect. Dis. 38 263-270 (2004)

226. Sawawiboon, N. et al. Lipodystrophy and reversal of facial lipoatrophy in perinatally HIV-infected children and adolescents after discontinuation of stavudine. Int. J. STD AIDS 23, 497-501 (2012).

227. Jagdeo, J., Ho, D., Lo, A. \& Carruthers, A. A systematic review of filler agents for aesthetic treatment of HIV facial lipoatrophy (FLA). J. Am. Acad. Dermatol. 73, 1040-1054.e14 (2015).

228. Duracinsky, M. et al. Safety of poly-L-lactic acid (New-Fills) in the treatment of facial lipoatrophy: a large observational study among HIV-positive patients. BMC Infect. Dis. 14, 474 (2014).

229. Bessesen, D. H. Update on obesity. J. Clin. Endocrinol. Metab. 93, 2027-2034 (2008).

230. Khera, R. et al. Association of pharmacological treatments for obesity with weight loss and adverse events: a systematic review and meta-analysis. JAMA 315, 2424-2434 (2016)

231. Pi-Sunyer, X. et al. A randomized, controlled trial of $3.0 \mathrm{mg}$ of liraglutide in weight management. N. Engl. J. Med. 373, 11-22 (2015).

232. Jia, X., Alam, M., Ye, Y., Bajaj, M. \& Birnbaum, Y GLP-1 receptor agonists and cardiovascular disease: a meta-analysis of recent cardiac outcome trials. Cardiovasc. Drugs Ther. 32, 65-72 (2018).

233. Kohli, R., Shevitz, A., Gorbach, S. \& Wanke, C. A randomized placebo-controlled trial of metformin for the treatment of HIV lipodystrophy. HIV Med. 8 420-426 (2007).

234. Mulligan, K. et al. The effects of recombinant human leptin on visceral fat, dyslipidemia, and insulin resistance in patients with human immunodeficiency virus-associated lipoatrophy and hypoleptinemia. J. Clin. Endocrinol. Metab. 94, 1137-1144 (2009).

235. Gagliano-Juca, T. \& Basaria, S. Testosterone replacement therapy and cardiovascular risk. Nat. Rev. Cardiol. 16, 555-574 (2019).

236. Bhasin, S. et al. Effects of testosterone supplementation on whole body and regional fat mass and distribution in human immunodeficiency virus-infected men with abdominal obesity. J. Clin. Endocrinol. Metab. 92 1049-1057 (2007).

237. Bredella, M. A. et al. Effects of $\mathrm{GH}$ on body composition and cardiovascular risk markers in young men with abdominal obesity. J. Clin. Endocrinol. Metab. 98, 3864-3872 (2013)

238. Bredella, M. A. et al. GH administration decreases subcutaneous abdominal adipocyte size in men with abdominal obesity. Growth Horm. IGF Res. 35, 17-20 (2017).

239. Bredella, M. A. et al. Effects of $\mathrm{GH}$ in women with abdominal adiposity: a 6-month randomized, doubleblind, placebo-controlled trial. Eur. J. Endocrinol. 166, 601-611 (2012).

240. Claessen, K. M. et al. Metabolic profile in growth hormone-deficient (GHD) adults after long-term recombinant human growth hormone ( $\mathrm{rhGH}$ ) therapy. J. Clin. Endocrinol. Metab. 98, 352-361 (2013)

241. Rochira, V. \& Guaraldi, G. Growth hormone deficiency and human immunodeficiency virus. Best. Pract. Res. Clin. Endocrinol. Metab. 31, 91-111 (2017). A study on growth hormone deficiency in PLWH.

242. Koutkia, P., Meininger, G., Canavan, B., Breu, J. \& Grinspoon, S. Metabolic regulation of growth hormone by free fatty acids, somatostatin, and ghrelin in HIV-lipodystrophy. Am. J. Physiol. Endocrinol. Metab. 286, E296-E303 (2004)

243. Rietschel, P. et al. Assessment of growth hormone dynamics in human immunodeficiency virus-related lipodystrophy. J. Clin. Endocrinol. Metab. 86, 504-510 (2001).

244. Kotler, D. P. et al. Effects of growth hormone on abnormal visceral adipose tissue accumulation and dyslipidemia in HIV-infected patients. J. Acquir. Immune Defic. Syndr. 35, 239-252 (2004).

245. Grunfeld, C. et al. Recombinant human growth hormone to treat HIV-associated adipose redistribution syndrome: 12 week induction and 24-week maintenance therapy. J. Acquir. Immune Defic. Syndr. 45, 286-297 (2007).

246. Stanley, T. L. \& Grinspoon, S. K. GH/GHRH axis in HIV lipodystrophy. Pituitary 12, 143-152 (2009).

247. Koutkia, P. et al. Growth hormone-releasing hormone in HIV-infected men with lipodystrophy: a randomized controlled trial. JAMA 292, 210-218 (2004).

248. Falutz, J. et al. A placebo-controlled, dose-ranging study of a growth hormone releasing factor in HIV-infected patients with abdominal fat accumulation. AIDS 19, 1279-1287 (2005).

249. Dhillon, S. Tesamorelin: a review of its use in the management of HIV-associated lipodystrophy. Drugs 71, 1071-1091 (2011).

250. Falutz, J. et al. Metabolic effects of a growth hormonereleasing factor in patients with HIV. N. Engl. J. Med. 357, 2359-2370 (2007)

A large controlled trial on the effect of tesamorelin on metabolic parameters in PLWH with truncal obesity

251. Falutz, J. et al. Effects of tesamorelin (TH9507), a growth hormone-releasing factor analog, in human immunodeficiency virus-infected patients with excess abdominal fat: a pooled analysis of two multicenter, double-blind placebo-controlled phase 3 trials with safety extension data. J. Clin. Endocrinol. Metab. 95 , 4291-4304 (2010).

252. Falutz, J. et al. Effects of tesamorelin, a growth hormone-releasing factor, in HIV-infected patients with abdominal fat accumulation: a randomized placebo-controlled trial with a safety extension J. Acquir. Immune Defic. Syndr. 53, 311-322 (2010).

253. Stanley, T. L. et al. Reduction in visceral adiposity is associated with an improved metabolic profile in HIV-infected patients receiving tesamorelin. Clin. Infect. Dis. 54, 1642-1651 (2012)

254. Stanley, T. L. et al. Effects of tesamorelin on inflammatory markers in HIV patients with excess abdominal fat: relationship with visceral adipose reduction. AIDS 25, 1281-1288 (2011).

255. Stanley, T. L. et al. Effect of tesamorelin on visceral fat and liver fat in HIV-infected patients with abdominal fat accumulation: a randomized clinical trial. JAMA 312, 380-389 (2014).

256. Fourman, L. T. et al. Visceral fat reduction with tesamorelin is associated with improved liver enzymes in HIV. AIDS 31, 2253-2259 (2017).

257. Stanley, T. L. et al. Effects of tesamorelin on non-alcoholic fatty liver disease in HIV: a randomised, double-blind, multicentre trial. Lancet HIV 6 , e821-e830 (2019).

258. Ekstedt, M. et al. Fibrosis stage is the strongest predictor for disease-specific mortality in NAFLD after up to 33 years of follow-up. Hepatology 61 , 1547-1554 (2015).

259. Clayton, P. E., Banerjee, I., Murray, P. G. \& Renehan, A. G. Growth hormone, the insulin-like growth factor axis, insulin and cancer risk. Nat. Rev. Endocrinol. 7, 11-24 (2011).

260. Popovic, V. et al. Serum insulin-like growth factor (IGF-I), IGF-binding proteins 2 and 3, and the risk for development of malignancies in adults with growth hormone $(\mathrm{GH})$ deficiency treated with $\mathrm{GH}$ : data from KIMS (Pfizer International Metabolic Database). J. Clin. Endocrinol. Metab. 95, 4449-4454 (2010).

261. Nguyen, N. T. \& Varela, J. E. Bariatric surgery for obesity and metabolic disorders: state of the art. Nat. Rev. Gastroenterol. Hepatol. 14, 160-169 (2017).

262. Akbari, K. et al. The effect of bariatric surgery on patients with HIV infection: a literature review. Obes. Surg. 28, 2550-2559 (2018).

263. Amouyal, C. et al. Sleeve gastrectomy in morbidly obese HIV patients: focus on anti-retroviral treatment absorption after surgery. Obes. Surg. 28, 2886-2893 (2018).

264. Lima, M. M. et al. Visceral fat resection in humans: effect on insulin sensitivity, beta-cell function, 
adipokines, and inflammatory markers. Obesity 21 E182-E189 (2013).

265. Herrera, M. F. et al. Potential additional effect of omentectomy on metabolic syndrome, acute-phase reactants, and inflammatory mediators in grade III obese patients undergoing laparoscopic Roux-en-Y gastric bypass: a randomized trial. Diabetes Care 33 1413-1418 (2010)

266. Brown, T. T. Approach to the human immunodeficiency virus-infected patient with lipodystrophy. J. Clin. Endocrinol. Metab. 93, 2937-2945 (2008).

267. Chen, S., Gui, X. E., Cao, Q. \& Routy, J. P. Clinical outcome after lipectomy in the management of patients with human immunodeficiency virusassociated dorsocervical fat accumulation: An observational cohort study. Medicine 98 e 16112 (2019)

268. Warren, A. G. \& Borud, L. J. Excisional lipectomy for HIV-associated cervicodorsal lipodystrophy. Aesthet. Surg. J. 28, 147-152 (2008).

269. UNAIDS. UNAIDS calls for greater urgency as globa gains slow and countries show mixed results towards 2020 HIV targets. UNAIDS https://www.unaids.org/en/ resources/presscentre/pressreleaseandstatementarchive 2019/july/20190716_PR_UNAIDS_global_report_2019 (2019).

270. Lazarus, J. V. et al. Beyond viral suppression of HIV - the new quality of life frontier. BMC Med. 14 , 94 (2016).

271. Erdbeer, G. et al. Everything fine so far? Physical and mental health in HIV-infected patients with virological success and long-term exposure to antiretroviral therapy. J. Int. AIDS Soc. 17, 19673 (2014).

272. Crane, H. M. et al. Lipoatrophy among HIV-infected patients is associated with higher levels of depression than lipohypertrophy. HIV Med. 9, 780-786 (2008).

273. Barlow-Mosha, L., Eckard, A. R., McComsey, G. A. \& Musoke, P. M. Metabolic complications and treatment of perinatally HIV-infected children and adolescents. J. Int. AIDS Soc. 16, 18600 (2013).

274. Miners, A. et al. Health-related quality-of-life of people with HIV in the era of combination antiretroviral treatment: a cross-sectional comparison with the general population. Lancet HIV 1, e32-e40 (2014).

275. Abel, G. \& Thompson, L. "I don't want to look like an AIDS victim": a New Zealand case study of facial lipoatrophy. Health Soc. Care Community 26, 41-47 (2018).

276. Guaraldi, G. et al. Nonalcoholic fatty liver disease in HIV-infected patients referred to a metabolic clinic: prevalence, characteristics, and predictors. Clin. Infect. Dis. 47, 250-257 (2008).

277. Ware, J. E., Jr. \& Sherbourne, C. D. The MOS 36-item short-form health survey (SF-36). I. Conceptual framework and item selection. Med. Care 30 , 473-483 (1992)

278. Wu, A. W., Revicki, D. A., Jacobson, D. \& Malitz, F. E. Evidence for reliability, validity and usefulness of the medical outcomes study HIV health survey (MOS-HIV). Qual. Life Res. 6, 481-493 (1997).
279. Blashill, A. J., Wilson, J. M., Baker, J. S., Mayer, K. H. \& Safren, S. A. Assessing appearance-related disturbances in HIV-infected men who have sex with men (MSM): psychometrics of the body change and distress questionnaire-short form (ABCD-SF). AIDS Behav. 18, 1075-1084 (2014).

280. Holmes, W. C. \& Shea, J. A. A new HIV/AIDS-targeted quality of life (HAT-QoL) instrument: development, reliability, and validity. Med. Care 36, 138-154 (1998).

281. Kraus, C. N., Chapman, L. W., Korta, D. Z. \& Zachary, C. B. Quality of life outcomes associated with treatment of human immunodeficiency virus (HIV) facial lipoatrophy. Int. J. Dermatol. $\mathbf{5 5}$, 1311-1320 (2016)

282. Leclercq, P. et al. High prevalence and impact on the quality of life of facial lipoatrophy and other abnormalities in fat tissue distribution in HIV-infected patients treated with antiretroviral therapy. AIDS Res. Hum. Retroviruses 29, 761-768 (2013).

283. Passos, S. M. \& Souza, L. D. An evaluation of quality of life and its determinants among people living with HIV/AIDS from Southern Brazil. Cad. Saude Publica 31, 800-814 (2015).

284. Verolet, C. M. et al. Lipodystrophy among HIV-infected patients: a cross-sectional study on impact on quality of life and mental health disorders. AIDS Res. Ther. 12, 21 (2015)

285. Adams, C., Stears, A., Savage, D. \& Deaton, C. "We're stuck with what we've got": the impact of lipodystrophy on body image. J. Clin. Nurs. 27, 1958-1968 (2018).

286. Wabe, N. T., Dekama, N. H. \& Gemeda, D. H. Lipodystrophy is common among Ethiopian patients on highly active antiretroviral therapy but is not associated with quality of life or medication adherence. Ther. Innov, Regul. Sci. 47, 706-714 (2013).

287. Casado, J. L. et al. Social isolation in HIV-infected patients according to subjective patient assessment and DEXA-confirmed severity of lipodystrophy. AIDS Care 25, 1599-1603 (2013).

288. Plankey, M. et al. The association of self-perception of body fat changes and quality of life in the women's interagency HIV study. AIDS Care 25, 1544-1550 (2013).

289. Lalanne, C. et al. Psychometric assessment of health-related quality of life and symptom experience in HIV patients treated with antiretroviral therapy. Qual. Life Res. 24, 1407-1418 (2015).

290. Hawkins, K. L. et al. Abdominal obesity, sarcopenia, and osteoporosis are associated with frailty in men living with and without HIV. AIDS 32, 1257-1266 (2018).

291. Rubin, L. H. et al. Midlife adiposity predicts cognitive decline in the prospective Multicenter AIDS Cohort Study. Neurology 93, e261-e271 (2019).

292. Sattler, F. R. et al. Abdominal obesity contributes to neurocognitive impairment in HIV-infected patients with increased inflammation and immune activation. J. Acquir. Immune Defic. Syndr. 68, 281-288 (2015).
293. Popkin, B. M. \& Slining, M. M. New dynamics in global obesity facing low- and middle-income countries. Obes. Rev. 14, 11-20 (2013).

294. Innes, S., Levin, L. \& Cotton, M. Lipodystrophy syndrome in HIV-infected children on HAART. South Afr. J. HIV Med. 10, 76-80 (2009).

295. Rydstrom, L. L., Wiklander, M., Naver, L., Ygge, B. M $\&$ Eriksson, L. E. HIV-related stigma and health-related quality of life among children living with HIV in Sweden. AIDS Care 28, 665-671 (2016).

296. Penazzato, M. et al. Optimizing research to speed up availability of pediatric antiretroviral drugs and formulations. Clin. Infect. Dis. 64, 1597-1603 (2017).

\section{Author contributions}

Introduction (J.C. and J.R.K.); Epidemiology (P.D., J.C. and J.E.L.); Mechanisms/pathophysiology (J.R.K. and C.L.); Diagnosis, screening and prevention (J.E.L.); Management (J.F.); Quality of life (A.C.); Outlook (T.T.B.); Overview of the primer (J.R.K. and J.C.). J.R.K. and C.L. contributed equally to the development of this Primer.

\section{Competing interests}

J.R.K. has received research support from Gilead Sciences and served as a consultant to Gilead Sciences and Theratechnologies. J.E L receives research support from Gilead Sciences and has served as a consultant to Gilead Sciences and Merck Sharp $\&$ Dohme. P.D. has received educational grants from and served as a consultant to Gilead Sciences, Johnson \& Johnson, Merck Sharp \& Dohme, and ViiV Healthcare. A.C. received unrestricted educational grants (to the institution) from BMS, Gilead, Merck Sharp \& Dohme and ViiV Healthcare. J.F. has served on speakers' bureaus from Gilead Sciences, Merck Sharp \& Dohme and ViiV Healthcare, and has served as a consultant to Theratechnologies up to 2015. T.T.B. has served as a consultant to Gilead Sciences, Merck, Theratechnologies and ViiV Healthcare. J.C. has received research support (to the institution) from Janssen, Merck Sharp \& Dohme and ViiV Healthcare, and educational grants from BMS, Gilead Sciences, Janssen, Merck Sharp \& Dohme and ViiV Healthcare. C.L. declares no competing interests.

\section{Peer review information}

Nature Reviews Disease Primers thanks J. Dave, G. Guaraldi and the other, anonymous, reviewer(s) for their contribution to the peer review of this work.

\section{Publisher's note}

Springer Nature remains neutral with regard to jurisdictional claims in published maps and institutional affiliations.

\section{RELATED LINKS}

90-90-90: treatment for all: https://www.unaids.org/en/ ources/909090

ClinicalTrials.gov: https://clinicaltrials.gov/

Theratechnologies: https://www.theratech.com

- Springer Nature Limited 2020 